\title{
Exponentially Stable Guaranteed Cost Control for Continuous and Discrete-time Takagi-Sugeno Fuzzy Systems
}

\author{
Bo Pang ${ }^{\mathrm{a}, \mathrm{b}}$, Xiaocheng Liu ${ }^{\mathrm{a}, \mathrm{b}}$, Qibing Jin ${ }^{\mathrm{c}}$, Weidong Zhang, \\ ${ }^{a}$ Department of Automation, Shanghai Jiao Tong University, Shanghai, 200240, PRC. \\ ${ }^{b}$ Key Laboratory of System Control and Information Processing, Ministry of Education, \\ Shanghai, 200240, PRC \\ ${ }^{c}$ Institute of Automation, Beijing University of Chemical Technology, Beisanhuan East \\ Road 15, Chaoyang District, Beijing, 100029, PRC.
}

\begin{abstract}
This paper investigates exponentially stable guaranteed cost control (GCC) for a class of nonlinear systems which is represented by Takagi-Sugeno (T-S) fuzzy systems. State feedback controllers of parallel distributed compensation (PDC) structure are designed by the means of GCC for continuous and discrete-time T-S fuzzy systems respectively. GCC methods in this paper adopt quadratic performance functions, which take effects of control effort$\mathrm{s}$, regulation errors and convergence rates into consideration simultaneously, to provide desirable performance and fast response for closed-loop systems. Sufficient design conditions that guarantee exponential stabilities of resulting closed-loop systems with predefined convergence rates are presented. By setting different convergence rates, response speed of the closed-loop nonlinear system can be adjusted. The proposed design procedures are eventually
\end{abstract}

\footnotetext{
*Corresponding author

Email address: wdzhang@sjtu.edu.cn (Weidong Zhang)
}

Preprint submitted to Neurocomputing

February 28, 2016

(C) 2016. This manuscript version is made available under the Elsevier user license http://www.elsevier.com/open-access/userlicense/1.0/ 
converted into linear matrix inequalities (LMIs) problems of minimizing upper bounds of the guaranteed cost functions. Finally, a well-known nonlinear benchmark control example and a truck-trailer example demonstrate the effectiveness and feasibility of all the proposed methods.

Keywords: Exponential stability, Convergence rate, Guaranteed cost control (GCC), T-S fuzzy system, Linear matrix inequality (LMI)

\section{Introduction}

In real world, it is not an easy task to design satisfactory controllers for complex systems due to serious inherent nonlinearities and parameter uncertainties in these systems. Although approximate nonlinear mathematical models could be established to describe parts of the nonlinearities and uncertainties, sometimes these models are too complicated to be tackled directly for controller design with existing mathematic tools. Moreover, in some cases limited knowledge and measurement to the system's mechanism even prevent people from obtaining appropriate nonlinear mathematical models. At this point, fuzzy technique provides one of powerful ways to reduce the difficulties, which has been successful in modeling and control of a wide range of various nonlinear systems, for example, non-adaptive fuzzy control [1, 2], adaptive fuzzy control $[3,4]$, etc. Among them, Takagi-Sugeno (T-S) fuzzy model which is firstly introduced in [5] have found popularity both in literature and industry since many kinds of nonlinear systems can be approximated by the model succinctly and human's experience could be incorporated in. T-S fuzzy model uses linear combinations of system inputs to form linear subsystems as their inference consequences and then nonlinearly synthesizes these 
subsystems to reconstruct the original system outputs. In this indirect way, T-S fuzzy model provides a powerful bridge connecting the actual nonlinear systems and classical linear systems so that the abundant theories and mature techniques in linear systems are possible to be applied, which mitigates the pain of designing controllers for nonlinear systems directly.

Many studies have been done in terms of applying linear control theories into T-S fuzzy models. However, most of existing works concern only the ultimate control performance, for example, stability, tracking, robustness and so on $[2,6,7,8,9]$. Little attention has been paid to transient control performance, such as response speed, settling time, overshoot, etc. In general, there are infinite controllers which guarantee stabilities of corresponding systems. It is necessary to select one controller which satisfies predefined transient performance from them. So it is interesting to figure out how to obtain a satisfactory controller under some specific transient performance for T-S fuzzy model.

One natural idea is to extend optimal control results of linear system to the T-S fuzzy model. By using the concept of PDC [1], locally optimal T-S fuzzy controllers were designed in [10] through solving standard LQR problem for each subsystems. If the synthesized controller satisfied the sufficient stability conditions for T-S models proposed in [2], a suboptimal controller was obtained, otherwise the design procedure repeated. Obviously this method is trial and error. By relaxing the Riccati equation constraint into inequality, guaranteed cost control (GCC) approach was achieved via LMIs through minimizing an upper bound of a quadratic performance measure in [11, 12]. Based on relaxed stability conditions [13], a globally suboptimal 
fuzzy controller was designed by minimizing the upper bound of a given global quadratic performance function [14]. In [15] a globally optimal and stable fuzzy controller design method for finite and infinite horizons was claimed to be found. However the controller may only be considered as suboptimal according to comments $[16,17]$.

In reality, in addition to stability and optimality, fast response is also a desired property for control systems. The time optimal control problem is formulated for this objective. Although it is well known that for some classes of nonlinear systems the time optimal control is a bang-bang control [18], this control strategy is rarely implemented in real systems due to the difficulties of characterizing the switching surface. Another concept related to fast response control is the convergence rate [19], which could be explained in the definition of exponential stability, a special case of asymptotic stability [20]. If the system state variable $x(t)$ satisfies

$$
\|x(t)\| \leq k\|x(0)\| e^{-\alpha t}, \quad \forall x(0) \in D_{0}, \quad \forall t>0
$$

where $D_{0}=\|x\|<r_{0}$ is a domain containing $x=0, \alpha, r_{0}$ and $k$ are positive constants, then the system is said to be exponentially stable and $\alpha$ is the convergence rate. Tanaka et.al proposed a fuzzy controller design method which can guarantee the predefined convergence rate of closed-loop system in [21]. By using LMI regions relevant to pole location constraints in [22], [23] presents a number of controller design methods for T-S fuzzy systems via $H^{\infty}$ optimization with pole assignment specifications.

However, all the aforementioned studies aim at either minimizing the standard quadratic performance function or achieving fast response. To author's best knowledge, few results which consider both the optimality and re- 
sponse speed at the same time are available for T-S fuzzy systems. Motived by above concerns, in this paper, we try to figure out an effective approach of GCC design with desirable convergence rate for a class of nonlinear systems represented by linear T-S fuzzy model. Firstly, the continuous-time modified quadratic cost function and its discrete counterpart are borrowed from classic linear system theories. Secondly, by utilizing the PDC concept, sufficient conditions are presented respectively for continuous- and discrete-time systems such that the designed controllers are able to exponentially stabilize the closed-loop system with predefined convergence rate and simultaneously minimize the upper bound of the cost functions. Thirdly, we show that the controller design problems can be transformed into LMI problems solved efficiently by available convex optimization tools. Finally, simulation results of two numerical examples are presented to illustrate the effectiveness and advantages of the proposed design methods. Compared with the previous literature, the main contributions of this paper are as follows.

1. By using the modified quadratic cost functions which not only represent the control effort and the regulation error but also take response speed into account [24], the proposed control method can provide closed-loop systems with ability of fast response while makes the cost functions minimized.

2. The relationship between response speed and convergence rate of the proposed control method is more intuitive than that between response speed and weighted matrices of classical GCC. By setting different convergence rates, the response speeds of closed-loop systems can be adjusted efficiently. 
Notation: Throughout this paper, if not explicitly stated, matrices are assumed to have compatible dimensions. "Continuous-time fuzzy systems" and "discrete-time fuzzy systems" are abbreviated as CFS and DFS, respectively. $A>0$ means that the matrix $A$ is positive definite; the notation $A>B$ means that $A-B>0$ is positive definite; $I$ is the identity matrix of appropriate dimension; 0 is the zero matrix of appropriate dimension; In the square matrix, the symbol ' $*$ ' stands for the transposed elements in the symmetric positions. In addition, $\sum_{i<j}^{r}$ and $\sum_{i \neq j}^{r}$ mean, for instance,

$$
\begin{gathered}
\sum_{i<j}^{3} x_{i j}=x_{12}+x_{23}+x_{13} \\
\sum_{i \neq j}^{3} x_{i j}=x_{12}+x_{13}+x_{21}+x_{23}+x_{31}+x_{32}
\end{gathered}
$$

\section{Problem formulation and preliminaries}

The T-S fuzzy models compose of a set of fuzzy "IF-THEN" rules with fuzzy sets in the antecedents and dynamic LTI systems in the consequents [5]. For a plant to be controlled, we can use the following sets of rules to describe it:

$i^{\text {th }}$ Plant Rule: IF $z_{1}(t)$ is $M_{i 1}, z_{2}(t)$ is $M_{i 2}, \cdots, z_{p}(t)$ is $M_{i p}$, THEN $\nabla x(t)=A_{i} x(t)+B_{i} u(t), y(t)=C_{i} x(t) . i=1,2, \cdots, r$.

where for expression simplicity, $t$ is used to represent either continuous or discrete time without causing ambiguity, $\nabla$ represents an operator. For CFS cases $\nabla x(t)$ means $\dot{x}(t)$ and for DFS cases $\nabla x(t)$ represents $x(t+1)[25]$. $x(t) \in R^{n}$ is the state vector, $u(t) \in R^{m}$ is the input vector, $y(t) \in R^{l}$ is the output vector, $A_{i} \in R^{n \times n}, B_{i} \in R^{n \times m}$ and $C_{i} \in R^{l \times n} . z(t) \in R^{p}$ is the 
premise variable vector, which is assumed to be independent of the input vector $u(t)$.

Each linear state equation in the consequent parts is called a "subsystem". By aggregating all the subsystems, the synthesized model is obtained as:

$$
\begin{gathered}
\nabla x(t)=\frac{\sum_{i=1}^{r} w_{i}(z(t))\left(A_{i} x(t)+B_{i} u(t)\right)}{\sum_{i=1}^{r} w_{i}(z(t))} \\
=\sum_{i=1}^{r} h_{i}(z(t))\left(A_{i} x(t)+B_{i} u(t)\right) \\
y(t)=\frac{\sum_{i=1}^{r} w_{i}(z(t)) C_{i} x(t)}{\sum_{i=1}^{r} w_{i}(z(t))}=\sum_{i=1}^{r} h_{i}(z(t)) C_{i} x(t)
\end{gathered}
$$

where

$$
\begin{aligned}
w_{i}(z(t)) & =\prod_{j=1}^{p} M_{i j}\left(z_{j}(t)\right) \geq 0 \\
Z(t) & =\left[\begin{array}{llll}
z_{1}(t) & z_{2}(t) & \cdots & z_{p}(t)
\end{array}\right] \\
h_{i}(z(t)) & =\frac{w_{i}(z(t))}{\prod_{i=1}^{r} w_{i}(z(t))}
\end{aligned}
$$

$M_{i j}\left(z_{j}(t)\right)$ is the grade of membership of $z_{j}(t)$ in the $j^{t h}$ fuzzy set $M_{i j}$ for all $t$. From (1) to (3), there exist

$$
\left\{\begin{array}{l}
1 \geq h_{i}(z(t)) \geq 0 \\
\sum_{i=1}^{r} h_{i}(z(t))=1
\end{array} \quad i=1,2, \cdots, r\right.
$$

The structure of the PDC controller for above fuzzy model is

$i^{\text {th }}$ Controller Rule: IF $z_{1}(t)$ is $M_{i 1}, z_{2}(t)$ is $M_{i 2}, \cdots, z_{p}(t)$ is $M_{i p}$, THEN $u_{i}(t)=-K_{i} x(t) . i=1,2, \cdots, r$.

where the antecedents are exactly the same with those of the fuzzy model and the consequents are linear state feedback laws similar to those in linear 
system theories. Aggregating all the controller rules, the synthesized fuzzy controller is represented by:

$$
u(t)=-\frac{\sum_{i=1}^{r} w_{i}(z(t)) K_{i} x(t)}{\sum_{i=1}^{r} w_{i}(z(t))}=-\sum_{i=1}^{r} h_{i}(z(t)) K_{i} x(t)
$$

Replacing (5) into (1), the closed-loop system is represented by:

$$
\begin{aligned}
\nabla x(t) & =\sum_{i=1}^{r} \sum_{j=1}^{r} h_{i}(z(t)) h_{j}(z(t))\left[A_{i}-B_{i} K_{j}\right] x(t) \\
& =\sum_{i=1}^{r} h_{i}^{2}(z(t)) G_{i i} x(t)+2 \sum_{i<j}^{r} h_{i}(z(t)) h_{j}(z(t)) \frac{G_{i j}+G_{j i}}{2} x(t)
\end{aligned}
$$

where

$$
G_{i j}=A_{i}-B_{i} K_{j}
$$

The purpose of this paper is to design a fuzzy state feedback controller (5) to exponentially stabilize the closed-loop T-S fuzzy system (6) and minimize the upper bound of corresponding performance function.

For positive definite matrix $Q \in R^{n \times n}$ and positive definite matrix $R \in$ $R^{m \times m}$, the following quadratic performance functions are considered [24]

$\langle\mathrm{CFS}\rangle$

$$
J_{c}=\int_{0}^{\infty} e^{2 \alpha t}\left[y^{T}(t) Q y(t)+u^{T}(t) R u(t)\right] d t
$$

$\langle\mathrm{DFS}\rangle$

$$
J_{d}=\sum_{t=0}^{\infty} e^{2 \alpha t T_{s}}\left[y^{T}(t) Q y(t)+u^{T}(t) R u(t)\right]
$$

where the convergence rate of the exponential function $\alpha>0$ reflects the requirement of response speed and $T_{s}$ is the sample time of DFS. Then the problem of exponentially stable GCC design can be formulated as follows 
Definition 1. Given a properly predefined real number $\alpha>0$, if there exist a state feedback PDC control law $u(t)$ in the form of (5), such that: (a) the closed-loop system (6) is exponentially stable with the convergence rate no less than $\alpha$; (b) the cost $J_{c}$ or $J_{d}$ of quadratic performance function (8) (or (9)) is no more than $J_{0}$. Then $u(t)$ is said to be exponentially stable GCC law for system (1) with (2) and $J_{0}$ is the guaranteed cost.

The stability conditions of fuzzy closed-loop control system (6) are derived using Lyapunov approach [13].

Lemma 1. (6) is asymptotically stable in the large if there exist a common matrix $P>0$ such that

$\langle C F S\rangle$

$$
\begin{array}{r}
G_{i i}^{T} P+P G_{i i}<0 \\
\left(\frac{G_{i j}+G_{j i}}{2}\right)^{T} P+P\left(\frac{G_{i j}+G_{j i}}{2}\right)<0
\end{array}
$$

$\langle D F S\rangle$

$$
\begin{array}{r}
G_{i i}^{T} P G_{i i}-P<0 \\
\left(\frac{G_{i j}+G_{j i}}{2}\right)^{T} P\left(\frac{G_{i j}+G_{j i}}{2}\right)-P<0
\end{array}
$$

for all $i$ and $j$ excepting the pairs $(i, j)$ such that $h_{i}(z(t)) h_{j}(z(t))=0, \forall t>0$.

Although Lemma 1 has guaranteed the stability of closed-loop systems, they may be quite conservative because no matter what the membership functions are, the conditions retain unchanged. Thus a relaxed stability condition is derived by considering the effect of membership functions as follows [13]. 
Lemma 2. Assume that the number of rules that fire for all t is no more than $s$, where $1<s \leq r$. The fuzzy closed-loop control system (6) is asymptotically stable in the large if there exist a common matrix $P>0$ and a common matrix $U_{0} \geq 0$ such that

$\langle C F S\rangle$

$$
\begin{array}{r}
G_{i i}^{T} P+P G_{i i}+(s-1) U_{0}<0 \\
\left(\frac{G_{i j}+G_{j i}}{2}\right)^{T} P+P\left(\frac{G_{i j}+G_{j i}}{2}\right)-U_{0}<0
\end{array}
$$

$\langle D F S\rangle$

$$
\begin{array}{r}
G_{i i}^{T} P G_{i i}-P+(s-1) U_{0}<0 \\
\left(\frac{G_{i j}+G_{j i}}{2}\right)^{T} P\left(\frac{G_{i j}+G_{j i}}{2}\right)-P-U_{0}<0
\end{array}
$$

for all $i$ and $j$ excepting the pairs $(i, j)$ such that $h_{i}(z(t)) h_{j}(z(t))=0, \forall t>0$.

The word 'fire' means that a rule's weight $h_{i}(z(t))$ for a given $z(t)$ in $(3)$ is not zero for all $t$. Noticing that for any $P$ satisfying Lemma 1 , there must exist some $U_{0}$ satisfying Lemma 2 , but it is not always the case vice versa. Thus the less conservative Lemma 2 contains more possible stable controller candidates and would give us more design choices. In the next section, main results of this paper are presented.

\section{Exponentially stable GCC design for CFS}

In this section, the sufficient conditions on the exponential stability with predefined convergence rate for CFS are provided by using the GCC approach and PDC concept. Then the design is transformed into a convex optimization represented by LMIs. 
Theorem 1. The system (1) with (2) for CFS case is exponentially stabilized with convergence rate no less than $\alpha>0$ by the PDC fuzzy controller (5) if there exist a common matrix $P>0$ and a common matrix $U_{0} \geq 0$ satisfying

$$
\begin{array}{r}
G_{i i}^{T} P+P G_{i i}+N_{i i}+(s-1) U_{0}+2 \alpha P<0 \quad \forall i \\
G_{i j}^{T} P+P G_{j i}+G_{j i}^{T} P+P G_{i j}+N_{i j}+N_{j i}-2 U_{0}+4 \alpha P<0 \quad i<j
\end{array}
$$

excepting the pairs $(i, j)$ such that $h_{i}(z(t)) h_{j}(z(t))=0, s>1, \forall t>0 . G_{i j}$ is as in (7) and

$$
N_{i j}=\left[\begin{array}{ll}
C_{i}^{T} & -K_{j}^{T}
\end{array}\right]\left[\begin{array}{ll}
Q & 0 \\
0 & R
\end{array}\right]\left[\begin{array}{c}
C_{i} \\
-K_{j}
\end{array}\right]
$$

Then the performance index (8) satisfies $J_{c}<x^{T}(0) P x(0)=J_{0}$.

Proof. To begin with, we derive an equivalent problem of the original exponentially stable GCC problem stated in Definition 1 by defining the following new variables

$$
\begin{array}{r}
x^{\alpha}(t)=e^{\alpha t} x(t), \quad u^{\alpha}(t)=e^{\alpha t} u(t) \\
\hat{y}^{\alpha}(t)=e^{\alpha t}\left[\begin{array}{c}
y(t) \\
u(t)
\end{array}\right]=\sum_{i=1}^{r} h_{i}(z(t))\left[\begin{array}{c}
C_{i} \\
-K_{i}
\end{array}\right] x^{\alpha}(t)
\end{array}
$$

Then cost function (8) could be rewritten as

$$
\begin{aligned}
J_{c} & =\int_{0}^{\infty}\left(\sum_{i=1}^{r} h_{i}(z(t))\left[\begin{array}{c}
C_{i} \\
-K_{i}
\end{array}\right] x^{\alpha}(t)\right)^{T}\left[\begin{array}{ll}
Q & 0 \\
0 & R
\end{array}\right] \sum_{i=1}^{r} h_{i}(z(t))\left[\begin{array}{c}
C_{i} \\
-K_{i}
\end{array}\right] x^{\alpha}(t) \mathrm{d} t \\
& =\int_{0}^{\infty}\left(\hat{y}^{\alpha}(t)\right)^{T}\left[\begin{array}{ll}
Q & 0 \\
0 & R
\end{array}\right] \hat{y}^{\alpha}(t) \mathrm{d} t
\end{aligned}
$$


and the new closed-loop system in terms of new variables is derived by using (1) and (5)

$$
\begin{aligned}
\dot{x}^{\alpha}(t) & =\alpha e^{\alpha t} x(t)+e^{\alpha t} \dot{x}(t) \\
& =\sum_{i=1}^{r} \sum_{j=1}^{r} h_{i}(z(t)) h_{j}(z(t))\left[\left(A_{i}-B_{i} K_{j}+\alpha I\right)\right] x^{\alpha}(t)
\end{aligned}
$$

Therefore through the definition of (21), the original problem regarding (6) and (8) is transformed to the equivalent problem regarding (23) and (22).

Now we prove the stability. Assuming that there are a common matrix $P>0$ and a common matrix $U_{0} \geq 0$ satisfying (18) and (19), and noticing that $Q>0, R>0$, we immediately obtain

$$
\begin{array}{r}
G_{i i}^{T} P+P G_{i i}+(s-1) U_{0}+2 \alpha P<0 \quad \forall i \\
G_{i j}^{T} P+P G_{i j}+G_{j i}^{T} P+P G_{j i}-2 U_{0}+4 \alpha P<0 \quad i<j
\end{array}
$$

which exactly shows that the fuzzy control system (23) is asymptotically stable in the large according to Lemma 2.

i.e.

$$
\lim _{t \rightarrow \infty} x^{\alpha}(t)=\lim _{t \rightarrow \infty} e^{\alpha t} x(t)=0
$$

which means that the original state $x(t)$ would convergence to zero at an exponential speed no less than $k\|x(0)\| e^{-\alpha t}, k$ is a positive constant. This implies that the original T-S fuzzy system (6) is exponentially stable.

Next, we prove that the quadratic performance function satisfies $J_{c}<$ $x^{T}(0) P x(0)$.

Consider the Lyapunov function $\left(x^{\alpha}(t)\right)^{T} P x^{\alpha}(t)$ and from (18), (19) and 
Corollary 2 in [13]

$$
\begin{aligned}
& \frac{\mathrm{d}}{\mathrm{d} t}\left(x^{\alpha}(t)\right)^{T} P x^{\alpha}(t)=\left(\dot{x}^{\alpha}(t)\right)^{T} P x^{\alpha}(t)+\left(x^{\alpha}(t)\right)^{T} P \dot{x}^{\alpha}(t) \\
& =\sum_{i=1}^{r} \sum_{j=1}^{r} h_{i}(z(t)) h_{j}(z(t))\left(x^{\alpha}(t)\right)^{T}\left[\left(A_{i}-B_{i} K_{j}+\alpha I\right)^{T} P\right. \\
& \left.+P\left(A_{i}-B_{i} K_{j}+\alpha I\right)\right] x^{\alpha}(t) \\
& =\sum_{i=1}^{r} h_{i}^{2}(z(t))\left(x^{\alpha}(t)\right)^{T}\left[G_{i i}^{T} P+P G_{i i}+2 \alpha P\right] x^{\alpha}(t) \\
& +\sum_{i<j}^{r} h_{i}(z(t)) h_{j}(z(t))\left(x^{\alpha}(t)\right)^{T}\left[G_{i j}^{T} P+P G_{i j}+G_{j i}^{T} P+P G_{j i}\right. \\
& \left.+4 \alpha P] x^{\alpha}(t)\right) \\
& <-\sum_{i=1}^{r} \sum_{j=1}^{r} h_{i}(z(t)) h_{j}(z(t))\left(x^{\alpha}(t)\right)^{T}\left[\begin{array}{ll}
C_{i}^{T} & -K_{i}^{T}
\end{array}\right]\left[\begin{array}{ll}
Q & 0 \\
0 & R
\end{array}\right] \\
& {\left[\begin{array}{c}
C_{j} \\
-K_{j}
\end{array}\right] x^{\alpha}(t)-\left[(s-1) \sum_{i=1}^{r} h_{i}^{2}(z(t))-2 \sum_{i<j}^{r} h_{i}(z(t)) h_{j}(z(t))\right]} \\
& x^{\alpha}(t)^{T} U_{0} x^{\alpha}(t) \leq-\left(\hat{y}^{\alpha}(t)\right)^{T}\left[\begin{array}{ll}
Q & 0 \\
0 & R
\end{array}\right] \hat{y}^{\alpha}(t)
\end{aligned}
$$

Therefore

$$
\frac{d}{d t}\left(x^{\alpha}(t)\right)^{T} P x^{\alpha}(t)<-\left(\hat{y}^{\alpha}(t)\right)^{T}\left[\begin{array}{ll}
Q & 0 \\
0 & R
\end{array}\right] \hat{y}^{\alpha}(t)
$$

Integrating both sides from 0 to $\infty$, we have

$$
J_{c}=\int_{0}^{\infty}\left(\hat{y}^{\alpha}(t)\right)^{T}\left[\begin{array}{ll}
Q & 0 \\
0 & R
\end{array}\right] \hat{y}^{\alpha}(t) d t<-\left.\left(x^{\alpha}(t)\right)^{T} P x^{\alpha}(t)\right|_{0} ^{\infty}
$$

since the fuzzy system (23) is stable

$$
J_{c}<\left(x^{\alpha}(0)\right)^{T} P x^{\alpha}(0)=x^{T}(0) P x(0)
$$


However in Theorem 1, the cost function $J_{c}$ depends on initial state conditions. To eliminate this matter, assume that the initial conditions are random variables with zero expectation and a covariance equal to identity matrix, i.e.

$$
\Xi\left[x_{0} x_{0}^{T}\right]=I \quad \Xi\left[x_{0}\right]=0
$$

where $\Xi$ is the expectation operator. Then the exponentially stable GCC design is transformed to be independent of initial conditions as stated in Corollary 1.

Corollary 1. The system (1) with (2) for CFS case is exponentially stabilized with convergence rate no less than $\alpha>0$ by the PDC fuzzy controller (5) if there exist a common matrix $P>0$ and a common matrix $U_{0} \geq 0$ satisfying (18) and (19).Then the expectation of the cost function (8) satisfies

$$
\Xi_{x_{0}} J_{c}=\Xi_{x_{0}} \int_{0}^{\infty} e^{2 \alpha t}\left[y^{T}(t) Q y(t)+u^{T}(t) R u(t)\right] d t<\operatorname{tr}(P)
$$

Proof. Notice that from (25) we have

$$
\Xi_{x_{0}}\left[x_{0}^{T} P x_{0}\right]=\operatorname{tr}(P)
$$

Then the procedure of proof is similar to Theorem 1.

In order to achieve as good control performance as possible, the cost function (8) needs to be minimized. Based on Corollary 1, the exponentially stable GCC design is formulated into an optimization problem of minimizing the upper bound of cost function in (26) by using LMI techniques in [26]. 
Theorem 2. For a given proper convergence rate $\alpha>0$, the PDC feedback control gain $K_{i}$ in each rule's consequent of the fuzzy control system (6) can be calculated by solving the following LMI problem

$$
\begin{array}{lr} 
& \min _{X, Y, M_{1}, \cdots, M_{r}} \operatorname{tr}(Z) \\
\text { s.t. } & X>0, \quad Y \geq 0 \\
& {\left[\begin{array}{cc}
Z & I \\
I & X
\end{array}\right]>0} \\
& U_{i i}<0, \quad \forall i \\
V_{i j}<0, \quad i<j
\end{array}
$$

excepting all the pairs $(i, j)$ such that $h_{i}(z(t)) h_{j}(z(t))=0, \forall t>0$ and $s>1$, where

$$
\begin{aligned}
& U_{i i}=\left[\begin{array}{ccc}
X A_{i}^{T}+A_{i} X-M_{i}^{T} B_{i}^{T}-B_{i} M_{i}+2 \alpha X+(s-1) Y & X C_{i}^{T} & -M_{i}^{T} \\
* & -Q^{-1} & 0 \\
* & * & -R^{-1}
\end{array}\right]
\end{aligned}
$$

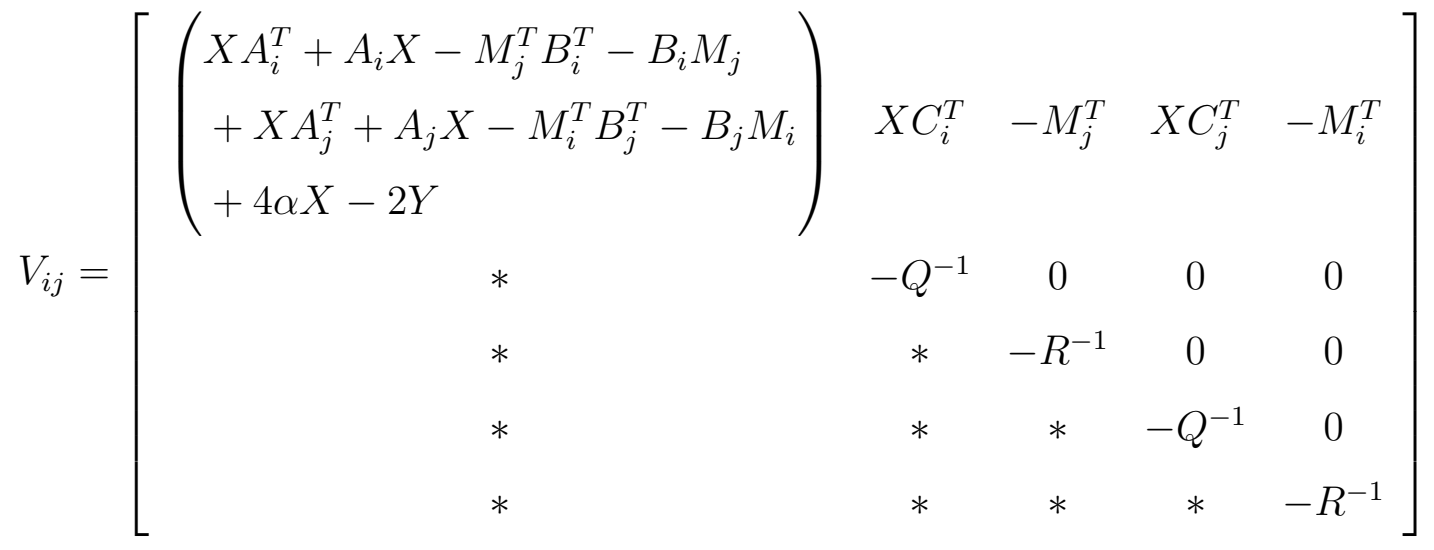

$$
X=P^{-1}, \quad M_{i}=K_{i} X, \quad Y=X U_{0} X
$$

If this problem is feasible, then the PDC feedback control gains can be obtained as $K_{i}=M_{i} X^{-1}$ for all $i$. 
Proof. By using Schur complement and congruent transformation in [26], conditions (28) and (29) follow easily from Theorem 1. The original design objective is to minimize the trace of $P$. However after variable transformation, we need to minimize the trace of $X^{-1}$, which is unsolvable by LMI technique. So the new variable $Z$ is introduced and let $Z-X^{-1}>0$, if it holds then $\operatorname{tr}(Z)>\operatorname{tr}\left(X^{-1}\right)=\operatorname{tr}(P)>J_{c}$. By using Schur complement, (27) is derived.

Remark 1. Theorem 1 and Corollary 1 provide sufficient conditions for the exponentially stable GCC designs. This design approach only requires to adjust one parameter $\alpha$, which is much more easier to guarantee the stability of the closed-loop system compared with the trial and error method in [10].

Remark 2. On one hand, it is interesting to find that, if all the terms regarding the quadratic performance parameter matrix $Q$ and $R$ in (18) and (19) are removed, then the stability sufficient conditions are exactly the Decay Rate Design in [21]. On the other hand, if convergence rate $\alpha$ is set to zero in (28) and (29), then the stability LMIs conditions are the same with those in [14]. Therefore, the approaches proposed in [21] and [14] are special cases of the method presented in this section, which synthesizes the respective advantages of two approaches and provides more design choices.

\section{Exponentially stable GCC design for DFS}

In this section, the sufficient conditions on the exponentially stability with predefined convergence rate are extended to discrete-time T-S fuzzy systems. 
Theorem 3. The system (1) with (2) for DFS case is exponentially stabilized with convergence rate no less than $\alpha>0$ by the PDC fuzzy controller (5) if there exist a common matrix $P>0$ and a common matrix $U_{0} \geq 0$ satisfying

$$
\begin{array}{r}
\left(G_{i i}^{\alpha}\right)^{T} P G_{i i}^{\alpha}-P+N_{i i}+(s-1) U_{0}<0 \quad \forall i \\
\left(\frac{G_{i j}^{\alpha}+G_{j i}^{\alpha}}{2}\right)^{T} P\left(\frac{G_{i j}^{\alpha}+G_{j i}^{\alpha}}{2}\right)-P+\frac{1}{2} N_{i j}+\frac{1}{2} N_{j i}-U_{0}<0 \quad i<j
\end{array}
$$

excepting the pairs $(i, j)$ such that $h_{i}(z(t)) h_{j}(z(t))=0, s>1, \forall t>0 . N_{i j}$ is as in (20) and

$$
\begin{array}{r}
G_{i j}^{\alpha}=A_{i}^{\alpha}-B_{i}^{\alpha} K_{j} \\
A_{i}^{\alpha}=e^{\alpha T_{s}} A_{i}, \quad B_{i}^{\alpha}=e^{\alpha T_{s}} B_{i}
\end{array}
$$

Then the performance index (9) satisfies $J_{d}<x^{T}(0) P x(0)=J_{0}$.

Proof. Similar to the proof of Theorem 1, an equivalent problem of the original fuzzy exponentially stable GCC problem stated in Definition 1 is derived by defining the new variables as follows

$$
\begin{array}{r}
x^{\alpha}(t)=e^{\alpha t T_{s}} x(t), \quad u^{\alpha}(t)=e^{\alpha t T_{s}} u(t)=-\sum_{i=1}^{r} h_{i}(z(t)) K_{i} x^{\alpha}(t) \\
\hat{y}^{\alpha}(t)=e^{\alpha t T_{s}}\left[\begin{array}{c}
y(t) \\
u(t)
\end{array}\right]=\sum_{i=1}^{r} h_{i}(z(t))\left[\begin{array}{c}
C_{i} \\
-K_{i}
\end{array}\right] x^{\alpha}(t)
\end{array}
$$

Then cost function (9) could be rewritten as

$$
\begin{aligned}
J_{d} & =\sum_{t=0}^{\infty}\left(\sum_{i=1}^{r} h_{i}(z(t))\left[\begin{array}{c}
C_{i} \\
-K_{i}
\end{array}\right] x^{\alpha}(t)\right)^{T}\left[\begin{array}{ll}
Q & 0 \\
0 & R
\end{array}\right] \sum_{i=1}^{r} h_{i}(z(t))\left[\begin{array}{c}
C_{i} \\
-K_{i}
\end{array}\right] x^{\alpha}(t) \\
& =\sum_{t=0}^{\infty}\left(\hat{y}^{\alpha}(t)\right)^{T}\left[\begin{array}{ll}
Q & 0 \\
0 & R
\end{array}\right] \hat{y}^{\alpha}(t)
\end{aligned}
$$


And the new closed-loop system in terms of new variables defined in (32) is derived by using (1) and (5)

$$
\begin{aligned}
x^{\alpha}(t+1) & =A_{i}^{\alpha} x^{\alpha}(t)+B_{i}^{\alpha} u^{\alpha}(t) \\
& =\sum_{i=1}^{r} \sum_{j=1}^{r} h_{i}(z(t)) h_{j}(z(t))\left[\left(A_{i}^{\alpha}-B_{i}^{\alpha} K_{j}\right)\right] x^{\alpha}(t)
\end{aligned}
$$

Therefore through the definition of (32) and (33), the original problem regarding (6) and (9) is transformed to the equivalent problem regarding (35) and (34).

Now we prove the stability. Assuming that there is a common matrix $P>0$ and a common matrix $U_{0} \geq 0$ satisfying (30) and (31), and noticing that $Q>0, R>0$, we immediately obtain

$$
\begin{array}{r}
\left(G_{i i}^{\alpha}\right)^{T} P G_{i i}^{\alpha}-P+(s-1) U_{0}<0 \quad \forall i \\
\left(\frac{G_{i j}^{\alpha}+G_{j i}^{\alpha}}{2}\right)^{T} P\left(\frac{G_{i j}^{\alpha}+G_{j i}^{\alpha}}{2}\right)-P-U_{0}<0 \quad i<j
\end{array}
$$

which exactly shows that the fuzzy control system (35) is asymptotically stable in the large according to Lemma 2.

i.e.

$$
\lim _{t \rightarrow \infty} x^{\alpha}(t)=\lim _{t \rightarrow \infty} e^{\alpha t T_{s}} x(t)=0
$$

which means that the original state $x(t)$ would convergence to zero at an exponential speed no less than $k\|x(0)\| e^{-\alpha t T_{s}}, k$ is a positive constant. This implies that the original T-S fuzzy system (6) is exponentially stable.

Next, we prove that the quadratic performance function satisfies $J_{d}<$ $x^{T}(0) P x(0)$.

Consider the Lyapunov function $V(x(t))=\left(x^{\alpha}(t)\right)^{T} P x^{\alpha}(t)$ and from (30), 
(31) and Corollary 2 in [13]

$$
\begin{aligned}
& \Delta V(x(t))=V(x(t+1))-V(x(t)) \\
& =\left(x^{\alpha}(t)\right)^{T} \frac{1}{4}\left[\sum_{i=1}^{r} \sum_{j=1}^{r} \sum_{k=1}^{r} \sum_{l=1}^{r} h_{i}(z(t)) h_{j}(z(t)) h_{k}(z(t)) h_{l}(z(t))\right. \\
& \left.\left[\left(G_{i j}^{\alpha}+G_{j i}^{\alpha}\right)^{T} P\left(G_{k l}^{\alpha}+G_{l k}^{\alpha}\right)-4 P\right] x^{\alpha}(t)\right] \\
& \leq \frac{1}{4} \sum_{i=1}^{r} \sum_{j=1}^{r} h_{i}(z(t)) h_{j}(z(t))\left(x^{\alpha}(t)\right)^{T}\left[\left(G_{i j}^{\alpha}+G_{j i}^{\alpha}\right)^{T} P\left(G_{i j}^{\alpha}+G_{j i}^{\alpha}\right)-4 P\right] x^{\alpha}(t) \\
& =\sum_{i=1}^{r} h_{i}^{2}(z(t))\left(x^{\alpha}(t)\right)^{T}\left[\left(G_{i i}^{\alpha}\right)^{T} P G_{i i}^{\alpha}-P\right] x^{\alpha}(t) \\
& +2 \sum_{i<j}^{r} h_{i}(z(t)) h_{j}(z(t))\left(x^{\alpha}(t)\right)^{T}\left[\left(\frac{G_{i j}^{\alpha}+G_{j i}^{\alpha}}{2}\right)^{T} P\left(\frac{G_{i j}^{\alpha}+G_{j i}^{\alpha}}{2}\right)-P\right] x^{\alpha}(t) \\
& <-\left(x^{\alpha}(t)\right)^{T} \sum_{i=1}^{r} \sum_{j=1}^{r} h_{i}(z(t)) h_{j}(z(t))\left[\begin{array}{ll}
C_{i}^{T} & -K_{i}^{T}
\end{array}\right]\left[\begin{array}{ll}
Q & 0 \\
0 & R
\end{array}\right]\left[\begin{array}{c}
C_{j} \\
-K_{j}
\end{array}\right] x^{\alpha}(t) \\
& -\left[(s-1) \sum_{i=1}^{r} h_{i}^{2}(z(t))-2 \sum_{i<j}^{r} h_{i}(z(t)) h_{j}(z(t))\right]\left(x^{\alpha}(t)\right)^{T} U_{0} x^{\alpha}(t) \\
& \leq-\left(\hat{y}^{\alpha}(t)\right)^{T}\left[\begin{array}{ll}
Q & 0 \\
0 & R
\end{array}\right] \hat{y}^{\alpha}(t)
\end{aligned}
$$

Therefore

$$
\Delta V(x(t))=V(x(t+1))-V(x(t))<-\left(\hat{y}^{\alpha}(t)\right)^{T}\left[\begin{array}{ll}
Q & 0 \\
0 & R
\end{array}\right] \hat{y}^{\alpha}(t)
$$

Accumulate both sides from $t=0$ to $\infty$ and noticing that the fuzzy system (35) is stable, we have 


$$
J_{d}=\sum_{t=0}^{\infty}\left(\hat{y}^{\alpha}(t)\right)^{T}\left[\begin{array}{ll}
Q & 0 \\
0 & R
\end{array}\right] \hat{y}^{\alpha}(t)<\left(x^{\alpha}(0)\right)^{T} P x^{\alpha}(0)=x^{T}(0) P x(0)
$$

Similarly, the Corollary 2 eliminates the effect of initial conditions on the design procedure.

Corollary 2. The system (1) with (2) for DFS case is exponentially stabilized with convergence rate no less than $\alpha>0$ by the PDC fuzzy controller (5) if there exist a common matrix $P>0$ and a common matrix $U_{0} \geq 0$ satisfying (30) and (31). Then the expectation of the cost function (9) satisfies

$$
\Xi_{x_{0}} J_{d}=\Xi_{x_{0}} \sum_{t=0}^{\infty} e^{2 \alpha t T_{s}}\left[y^{T}(t) Q y(t)+u^{T}(t) R u(t)\right]<\operatorname{tr}(P)
$$

Proof. The proof is trivial according to Corollary 1.

Similar to last section, we have following LMI optimization problem to achieve better control performance.

Theorem 4. For a given proper convergence rate $\alpha>0$, the PDC feedback control gains $K_{i}$ in each rule's consequent of the fuzzy control system (6) can be calculated by solving the following LMI problem

$$
\begin{array}{lr} 
& \min _{X, Y, M_{1}, \cdots, M_{r}} \operatorname{tr}(Z) \\
\text { s.t. } & X>0, \quad Y \geq 0 \\
& {\left[\begin{array}{rr}
Z & I \\
I & X
\end{array}\right]>0} \\
& U_{i i}<0, \quad \forall i \\
V_{i j}<0, \quad i<j
\end{array}
$$


excepting all the pairs $(i, j)$ such that $h_{i}(z(t)) h_{j}(z(t))=0, \forall t>0$ and $s>1$, where

$$
\begin{aligned}
& U_{i i}=\left[\begin{array}{cccc}
(s-1) Y-X & \left(X\left(A_{i}^{\alpha}\right)^{T}-M_{i}^{T}\left(B_{i}^{\alpha}\right)^{T}\right) & X C_{i}^{T} & -M_{i}^{T} \\
* & -X & 0 & 0 \\
* & * & -Q^{-1} & 0 \\
* & * & * & -R^{-1}
\end{array}\right]
\end{aligned}
$$

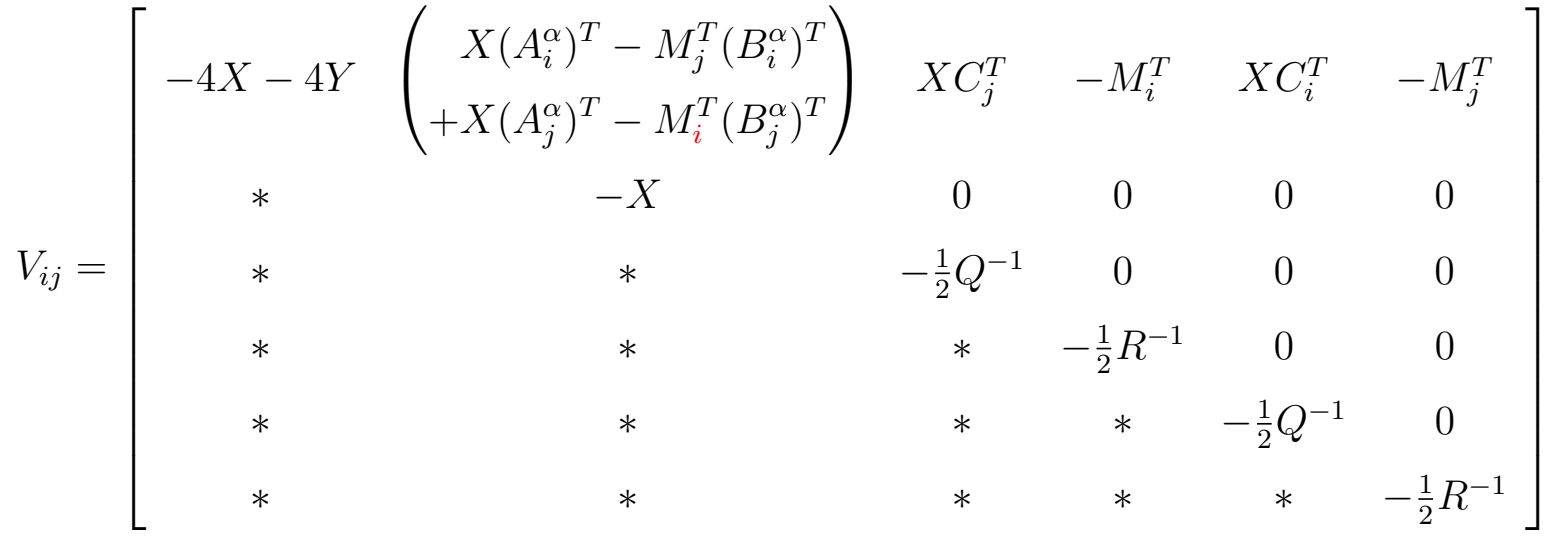

$$
\begin{aligned}
& X=P^{-1}, \quad M_{i}=K_{i} X, \quad Y=X U_{0} X
\end{aligned}
$$

If this problem is feasible, then the PDC feedback control gains can be obtained as $K_{i}=M_{i} X^{-1}$ for all $i$.

Proof. By using Schur complement and congruent transformation in [26], conditions (39) and (40) follow easily from Theorem 3. (38) is derived in the same way with $(27)$.

\section{Numerical Examples}

In this section, two numerical examples are simulated to demonstrate the effectiveness and feasibility of the proposed methods in section 3 and section 4 respectively. All simulations are performed on MATLAB 2012a with the 
convex optimization tools MOSEK [27] and YALMIP [28]. Some comparisons with other methods are presented to show the merits of our methods.

Example 1: Figure 1 shows a transitional oscillator with an eccentric rotational proof mass actuator (TORA) system, which is a well-known nonlinear benchmark control problem [29] and has been studied and reported in $[11,14,30,31,32]$. As Figure 1 illustrates, the nonlinear coupling between the rotational motion of the actuator and the translational motion of the oscillator provides the mechanism for control. After simplification, the system is described by the non-dimensional equations

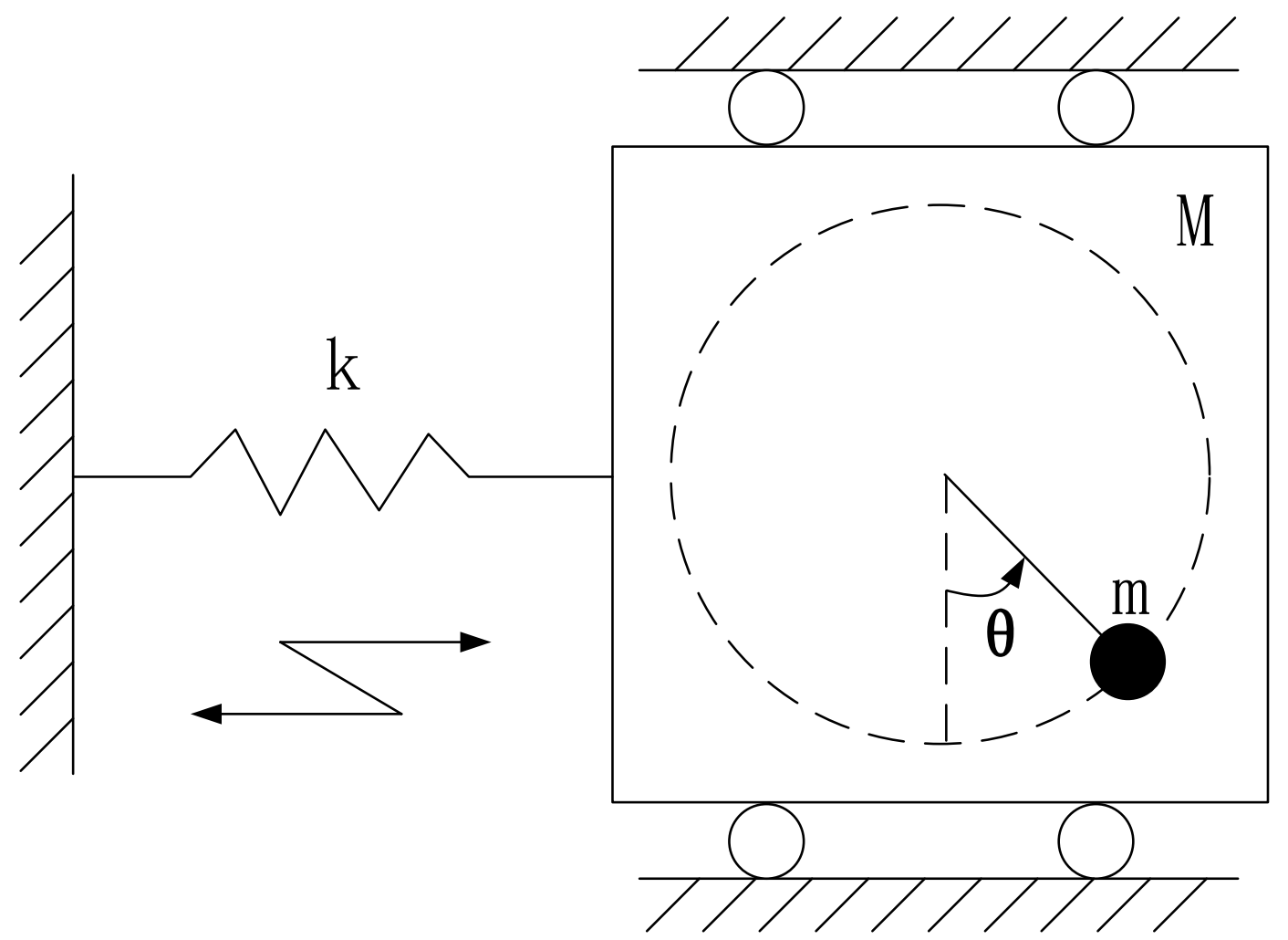

Figure 1: TORA system 


$$
\begin{array}{r}
\dot{x}(t)=f(x)+g(x) u(t)+d(x) w(t) \\
\left.f(x)=\left[\begin{array}{c}
x_{2} \\
\frac{\epsilon x_{4}^{2} \sin \left(x_{3}\right)-x_{1}}{1-\epsilon^{2} \cos ^{2}\left(x_{3}\right)} \\
x_{4} \\
g(x)=\left[\begin{array}{c}
0 \\
\frac{-\epsilon \cos \left(x_{3}\right)\left(x_{1}-\epsilon x_{4}^{2} \sin \left(x_{3}\right)\right)}{1-\epsilon^{2} \cos ^{2}\left(x_{3}\right)}
\end{array}\right] \\
\frac{1}{1-\epsilon^{2} \cos ^{2}\left(x_{3}\right)} \\
0 \\
\frac{1}{1-\epsilon^{2} \cos ^{2}\left(x_{3}\right)}
\end{array}\right], \quad \begin{array}{c}
0 \\
\frac{1}{1-\epsilon^{2} \cos ^{2}\left(x_{3}\right)} \\
0 \\
\frac{-\epsilon \cos ^{2}\left(x_{3}\right)}{1-\epsilon^{2} \cos ^{2}\left(x_{3}\right)}
\end{array}\right]
\end{array}
$$

where $u(t)$ is the control input, $w(t)$ is the disturbance input. $x_{1}$ and $x_{2}$ denote the transitional position and velocity of the cart with $\dot{x}_{1}=x_{2} \cdot x_{3}=\theta$ and $x_{4}=\dot{x}_{3}$ denote the angular position and velocity of the rotational proof mass.

To obtain the T-S fuzzy model of TORA for control design from (41), it is a simple and convenient way to linearize the system at local working points and then blend the local subsystems with properly designed membership functions. Commonly the more local working points the original system is linearized at, the more precise the resulting T-S fuzzy model is. However, simulation results here will demonstrate that even if only two working points are linearized for control design, the original TORA is still well controlled. By calculating the Jacobian of system (41) at points $\theta=0^{\circ}$ and $80^{\circ}$, resulting T-S fuzzy model is defined by the rules

Plant Rule 1: IF $x_{3}(t)$ is $M_{1}$, THEN $\dot{x}(t)=A_{1} x(t)+B_{1} u(t), y(t)=C_{1} x(t)$ Plant Rule 2: IF $x_{3}(t)$ is $M_{2}$, THEN $\dot{x}(t)=A_{2} x(t)+B_{2} u(t), y(t)=C_{2} x(t)$ 
where $M_{1}$ and $M_{2}$ are input fuzzy sets defined by membership functions

$$
\mu_{1}\left(x_{3}(t)\right)=1-\frac{2}{\pi}\left|x_{3}(t)\right|, \quad \mu_{2}\left(x_{3}(t)\right)=\frac{2}{\pi}\left|x_{3}(t)\right|
$$

respectively, other parameters are given as follows

$$
\begin{array}{ll}
A_{1}=\left[\begin{array}{cccc}
0 & 1 & 0 & 0 \\
-\frac{1}{1-\epsilon^{2}} & 0 & 0 & 0 \\
0 & 0 & 0 & 1 \\
\frac{\epsilon}{1-\epsilon^{2}} & 0 & 0 & 0
\end{array}\right], \quad B_{1}=\left[\begin{array}{c}
0 \\
-\frac{\epsilon}{1-\epsilon^{2}} \\
0 \\
\frac{1}{1-\epsilon^{2}}
\end{array}\right], \quad C_{1}=\left[\begin{array}{llll}
1 & 0 & 0 & 0 \\
0 & 1 & 0 & 0 \\
0 & 0 & 1 & 0 \\
0 & 0 & 0 & 1
\end{array}\right] \\
A_{2}=\left[\begin{array}{cccc}
0 \\
-\frac{1}{1-\epsilon^{2} \beta^{2}} & 0 & 0 & 0 \\
0 & 0 & 0 & 1 \\
\frac{\epsilon \beta}{1-\epsilon^{2} \beta^{2}} & 0 & 0 & 0
\end{array}\right], \quad B_{2}=\left[\begin{array}{c}
0 \\
-\frac{\epsilon \beta}{1-\epsilon^{2} \beta^{2}} \\
0 \\
\frac{1}{1-\epsilon^{2} \beta^{2}},
\end{array}\right], \quad C_{2}=C_{1}
\end{array}
$$

where $\beta=\cos \left(80^{\circ}\right)$.

Let $\epsilon=0.5$ and $w(t)=0$. The weighting matrices in quadratic performance (8) are chosen as

$$
Q=\left[\begin{array}{cccc}
0.5 & 0 & 0 & 0 \\
0 & 0.5 & 0 & 0 \\
0 & 0 & 1 & 0 \\
0 & 0 & 0 & 1
\end{array}\right] \times 10^{-4}, R=10^{-8}
$$

By using Theorem 2 for fuzzy GCC design in section 3 and choosing the convergence rate $\alpha=0.1$, resulting state feedback controller gains are

$$
\begin{aligned}
& K_{1}=[-4.7826,0.0061,0.5379,1.8669] \\
& K_{2}=[-4.7272,-0.9135,0.4816,1.7448]
\end{aligned}
$$


The matrices $X$ and $Y$ are

$$
\begin{aligned}
& X=\left[\begin{array}{cccc}
0.0507 & -0.0168 & -0.0037 & -0.0133 \\
-0.0168 & 0.0292 & 0.0030 & 0.0082 \\
-0.0037 & 0.0030 & 0.0008 & 0.0018 \\
-0.0133 & 0.0082 & 0.0018 & 0.0054
\end{array}\right] \\
& Y=\left[\begin{array}{cccc}
0.0571 & 0.0008 & -0.1333 & 0.0187 \\
0.0008 & 0.0005 & 0.0041 & -0.0044 \\
-0.1333 & 0.0041 & 0.4768 & -0.1401 \\
0.0187 & -0.0044 & -0.1401 & 0.0723
\end{array}\right]
\end{aligned}
$$

with $J_{0}=0.0861$.

For comparison, the controller based on standard quadratic performance function (QPF) in [14], the model-based GCC (MB-GCC) controller in [11] and the decay rate design (DRD) in [21] are also applied to the system with the same settings of $Q, R$ and $\alpha$ (if needed). Figure 2 to Figure 7 show the performance comparisons and control effort comparisons of the resulting simulation with initial condition $x_{0}=\left[\begin{array}{llll}0.3 & 0 & -40^{\circ} & 0\end{array}\right]^{T}$. 

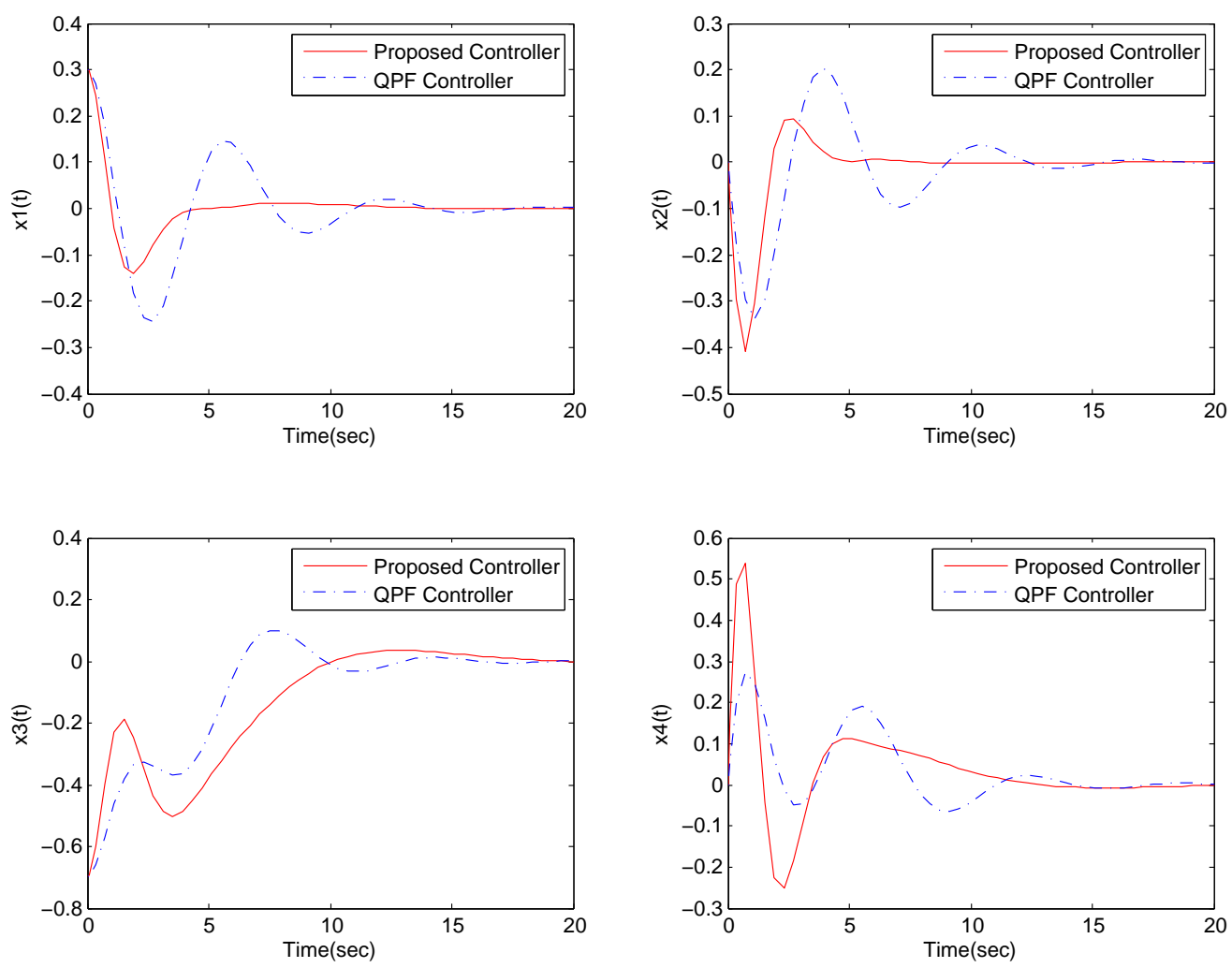

Figure 2: Performance comparison of QPF controller and the proposed 


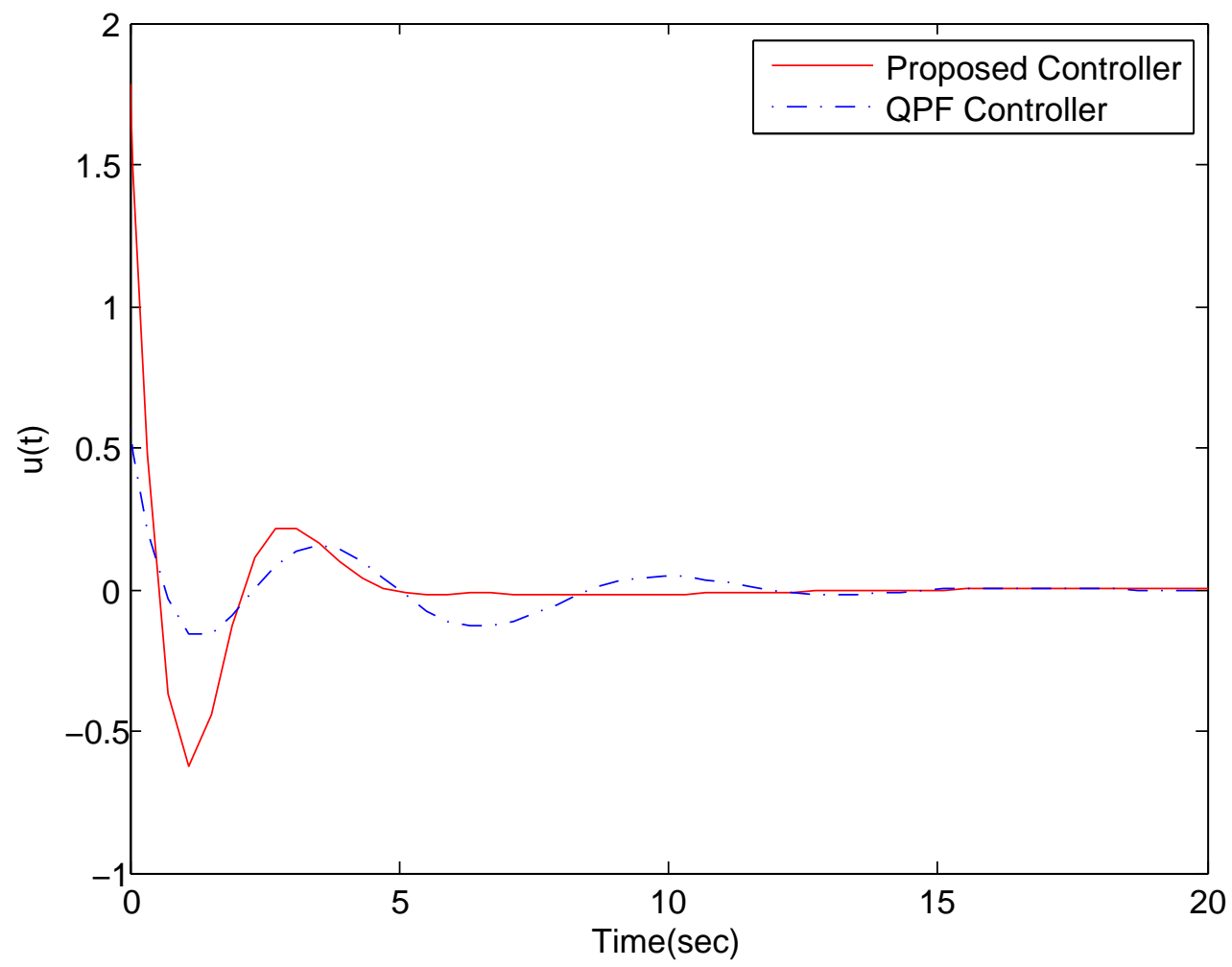

Figure 3: Control effort comparison of QPF controller and the proposed 

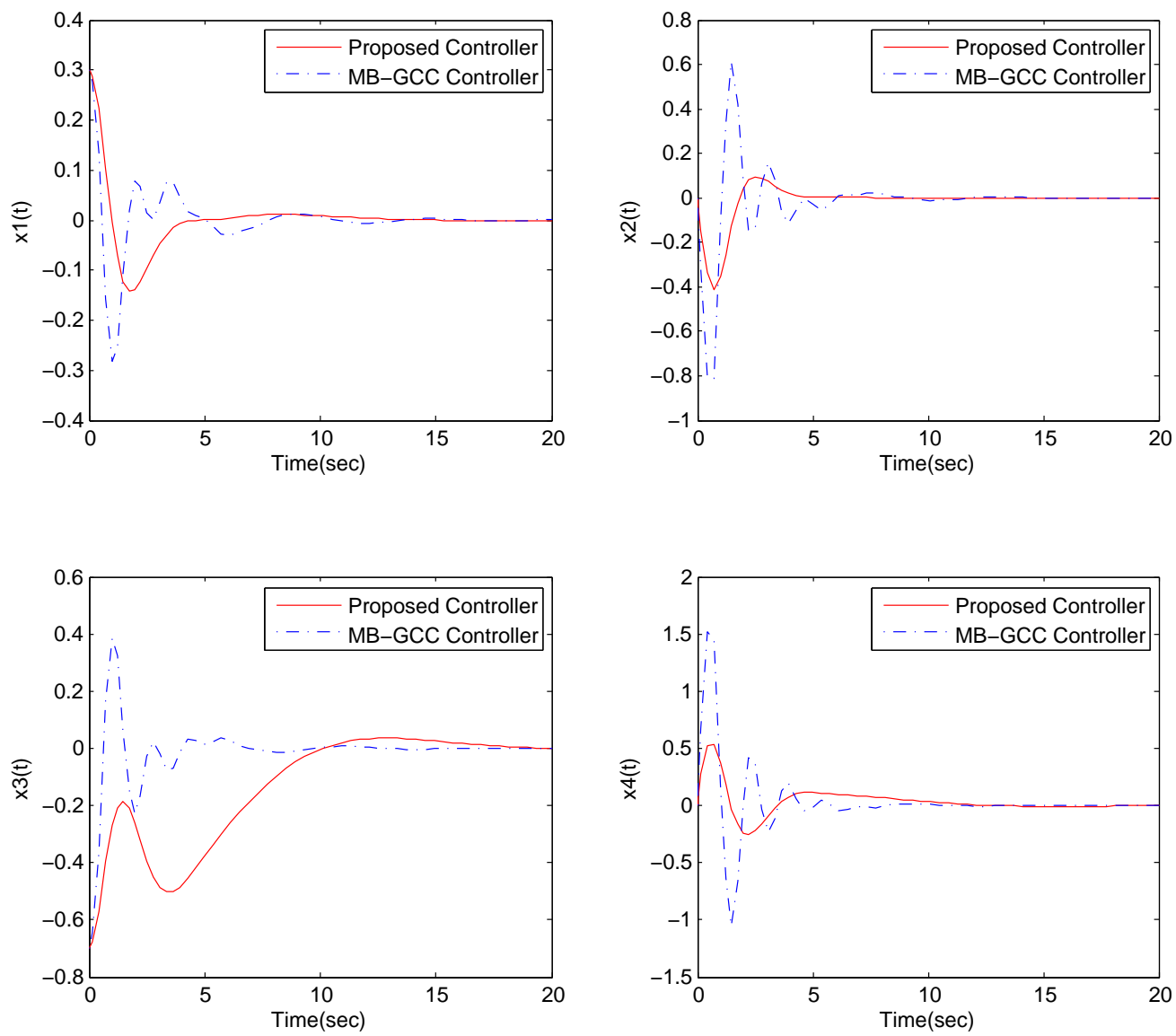

Figure 4: Performance comparison of MB-GCC controller and the proposed 


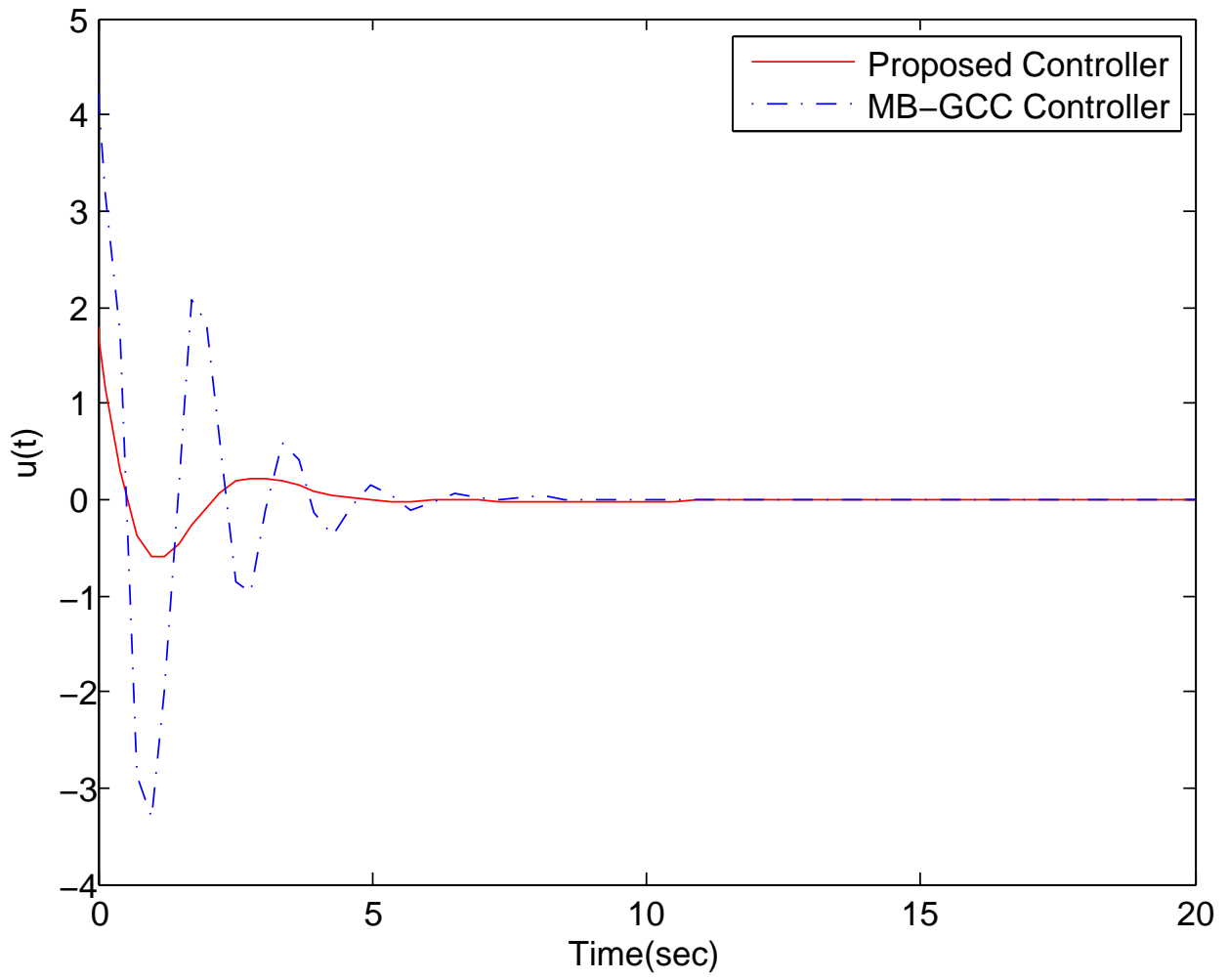

Figure 5: Control effort comparison of MB-GCC controller and the proposed 

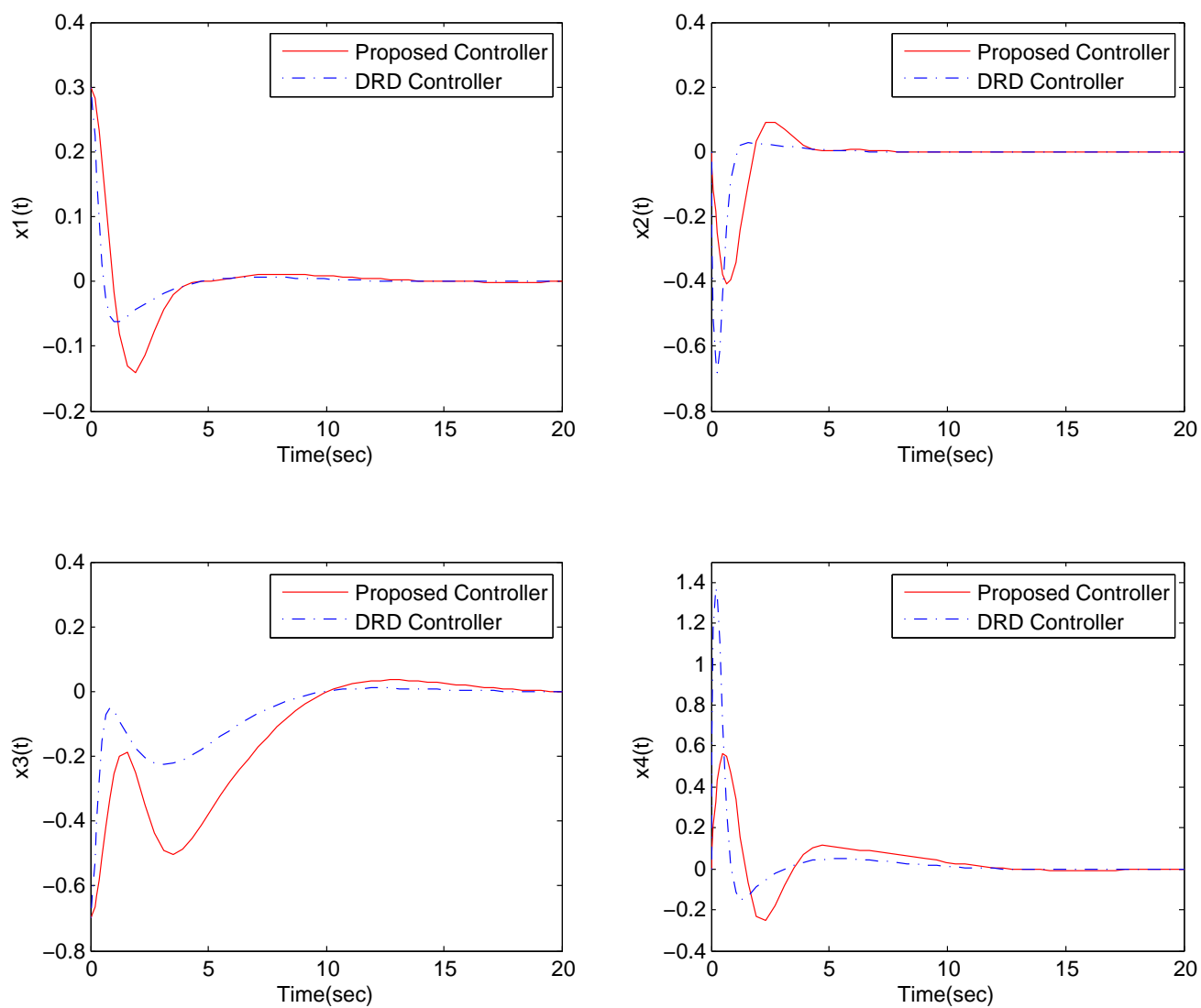

Figure 6: Performance comparison of DRD controller and the proposed 


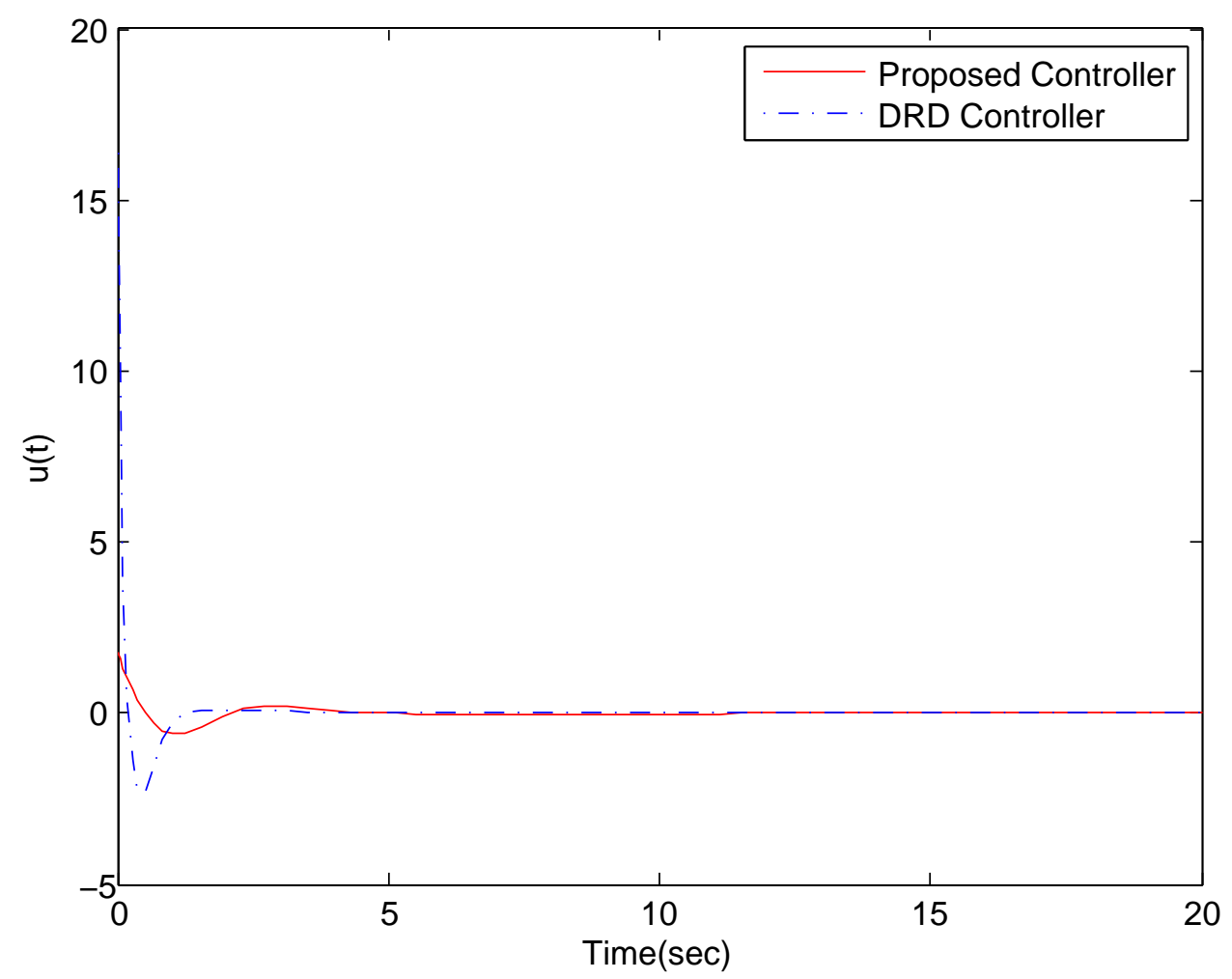

Figure 7: Control effort comparison of DRD controller and the proposed

From Figure 2 and Figure 3, one can find that the responses with proposed controller is faster and milder than those with QPF controller while the control effort with proposed controller is larger than that with QPF controller. As stated in the Remark 2, the method in [14] is equivalent to the proposed method when $\alpha=0$. Thus it is reasonable that the faster response the control task requires, the much control effort the system needs. The utilization of quadratic performance function (8) affords a trade-off between the convergence rate and the control effort such that the control effort would not become too large. This kind of trade-off is the very advantage of our 
method.

For Figure 4 and Figure 5, it is observed that though response speeds are similar in both cases, the transient responses with proposed controller are generally superior than those with MB-GCC Controller while the control effort with proposed controller is smaller than that of MB-GCC controller.

Figure 6 and Figure 7 show that the responses with proposed controller are generally milder than those with DRD controller although the response with DRD controller is a little faster than that with proposed controller. Therefore, performance of two controller can be evaluated as almost same. However, the control effort with DRD controller is much larger than that with proposed controller. Thus, the overall performance of DRD controller is inferior to that of proposed controller.

To sum up, the proposed controller generally provides better performance than the other three ones do.

Remark 3. In above simulation, the range of convergence rate $\alpha$ in which our method has feasible solutions is approximately [0,0.224]. It should be pointed out that the approaches in [11] and [14] can provide only one controller (if the problem is feasible) for a given T-S fuzzy system and a given pair of $Q$ and $R$ while our method has the potential to provide more possible controllers by setting different $\alpha>0$.

Figure 8 show responses of state $x_{1}(t)$ under proposed control with different values of $\alpha$. One can find that as $\alpha$ increases, the response speed of the original TORA system increases efficiently. 


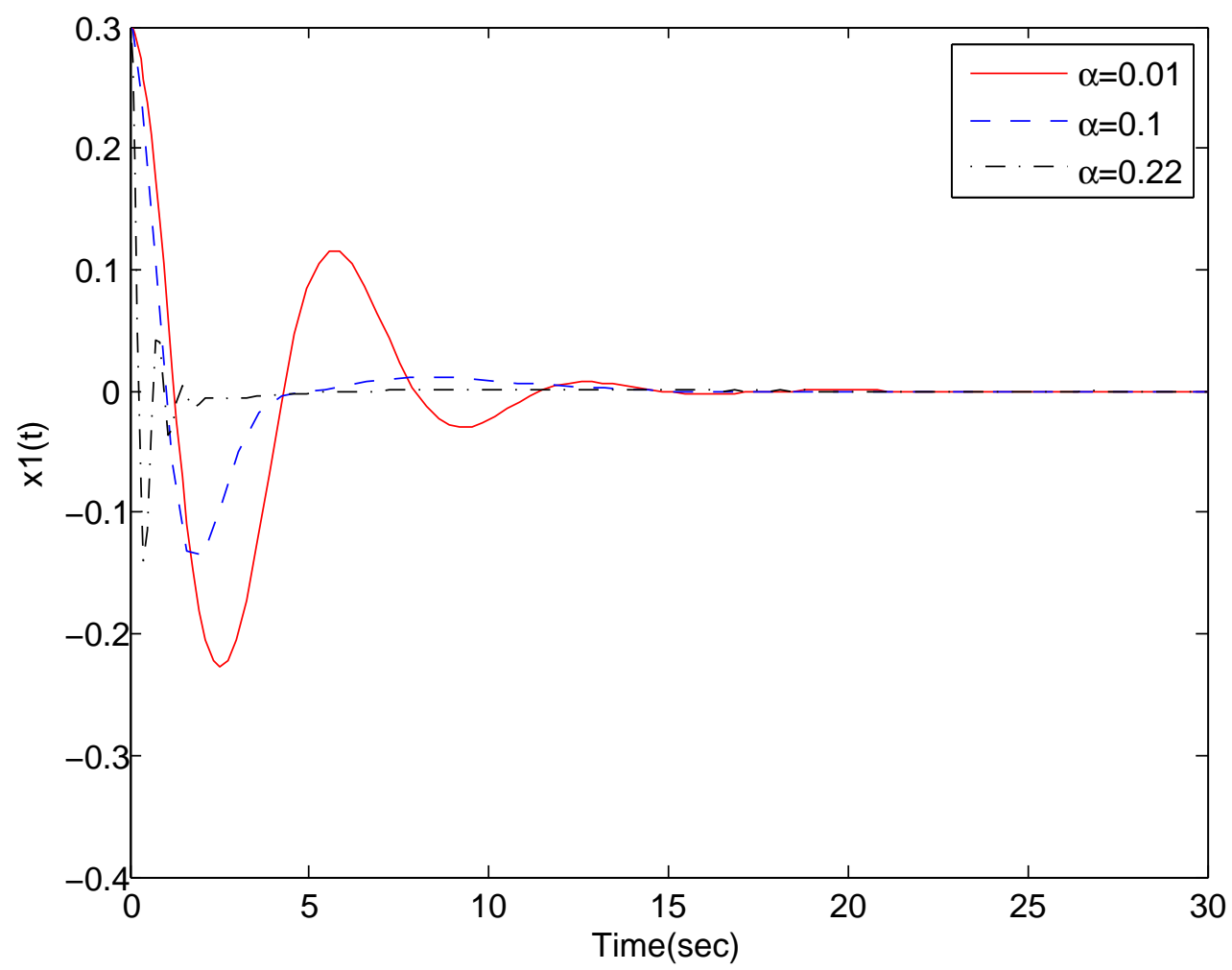

Figure 8: Responses comparison of the translational position $x_{1}(t)$ of the cart for TORA system with different $\alpha$ under proposed control

Remark 4. In fact, the weighting matrices $Q$ and $R$ can also affect the response speed of closed-loop systems. However, compared with the much more intuitive relationship between $\alpha$ and resulting response speed in our method, the relationship between response speed and weighting matrices is too obscure to be utilized for adjusting response speed effectively, .

In order to test the robustness of our proposed method, disturbance is added to the system with $w(t)=\sin (20 t)$. Figure 9 shows the response of each state. The proposed methods can stabilize the original TORA system 
in the presence of disturbances.
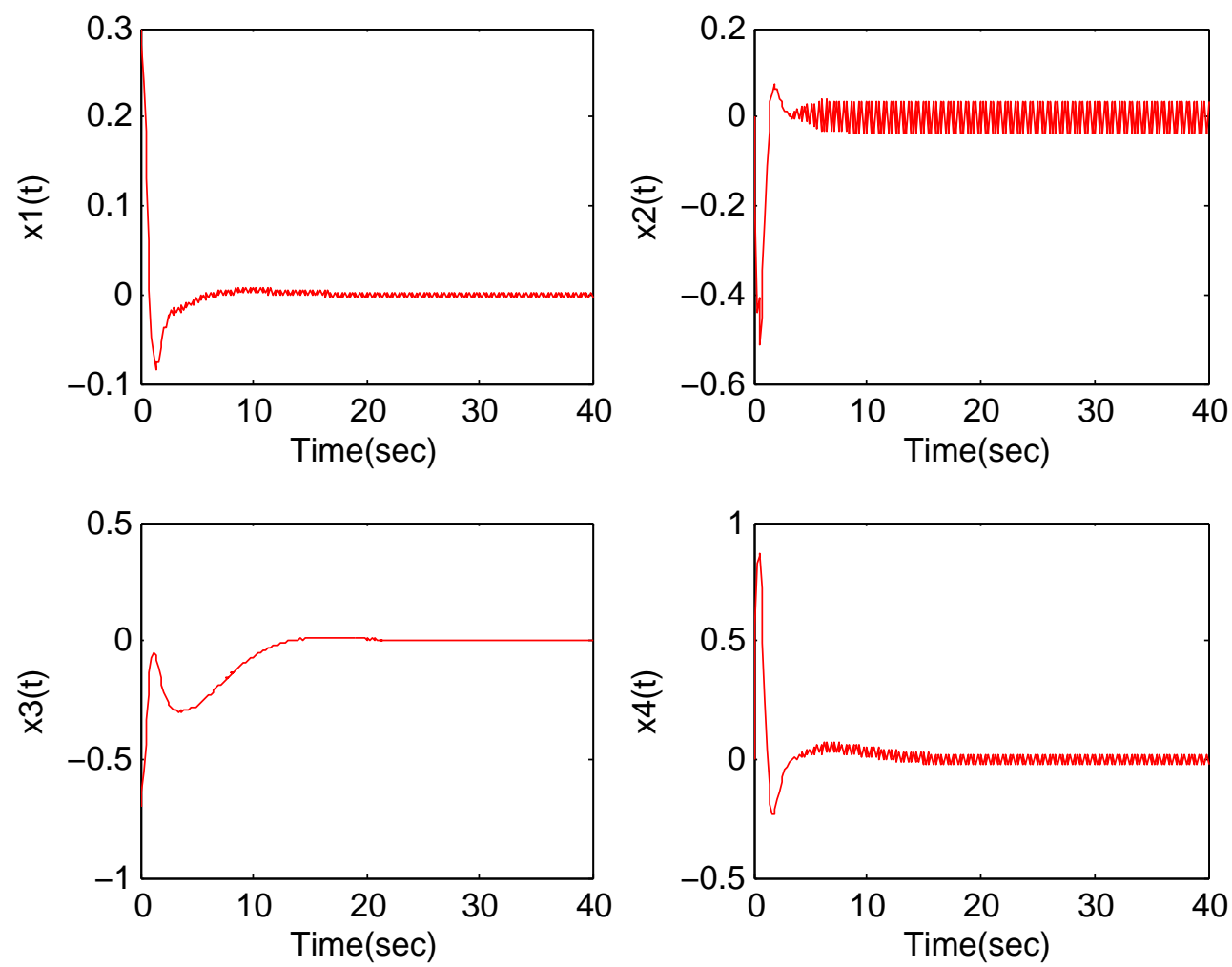

Figure 9: Responses of TORA system with sinusoidal disturbance

Example 2: This example will demonstrate the feasibility and efficiency of exponentially stable GCC design for DFS in section 4. Consider the backingup control of a computer-simulated truck-trailer which is borrowed from [33, 34]. The truck-trailer model is formulated as

$$
\begin{aligned}
& x_{1}(t+1)=\left(1-\frac{v t}{L}\right) x_{1}(t)+\frac{v t}{l} u(t) \\
& x_{2}(t+1)=x_{2}(t)+\frac{v t}{L} x_{1}(t) \\
& x_{3}(t+1)=x_{3}(t)+v t \sin \left[x_{2}(t)+\frac{v t}{2 L} x_{1}(t)\right]
\end{aligned}
$$


The following T-S fuzzy models are used to design the fuzzy controller

$$
\begin{aligned}
& \text { Plant Rule 1: IF } \theta(t)=x_{2}(t)+\frac{v t}{2 L} x_{1}(t) \quad \text { is about } 0, \\
& \text { THEN } x(t+1)=A_{1} x(t)+B_{1} u(t), y(t)=C_{1} x(t)
\end{aligned}
$$

Plant Rule 2: IF $\theta(t)=x_{2}(t)+\frac{v t}{2 L} x_{1}(t)$ is about $\pi$ or $-\pi$,

$$
\text { THEN } x(t+1)=A_{2} x(t)+B_{2} u(t), y(t)=C_{2} x(t)
$$

$$
\begin{array}{rlrl}
A_{1} & =\left[\begin{array}{ccc}
1-\frac{v \bar{t}}{L} & 0 & 0 \\
\frac{v \bar{t}}{L} & 1 & 0 \\
\frac{v^{2} \bar{t}^{2}}{2 L} & v \bar{t} & 1
\end{array}\right], & B_{1}=\left[\begin{array}{c}
\frac{v \bar{t}}{l} \\
0 \\
0
\end{array}\right], & C_{1}=\left[\begin{array}{lll}
1 & 0 & 0 \\
0 & 1 & 0 \\
0 & 0 & 1
\end{array}\right] \\
A_{2}=\left[\begin{array}{ccc}
1-\frac{v \bar{t}}{L} & 0 & 0 \\
\frac{v \bar{t}}{L} & 1 & 0 \\
\frac{d v^{2} \bar{t}^{2}}{2 L} & d v \bar{t} & 1
\end{array}\right], & B_{2}=\left[\begin{array}{c}
\frac{v \bar{t}}{l} \\
0 \\
0
\end{array}\right], & C_{2}=C_{1}
\end{array}
$$

where the length of truck is $l=2.8$, the length of trailer is $L=5.5$, the constant speed of backing up is $v=-1.0$, the sampling time is $\bar{t}=2.0$, $d=(0.01 / \pi)$ and the membership functions are

$$
\mu_{1}(\theta(t))=\frac{\sin (\theta(t))}{\theta(t)}, \quad \mu_{2}(\theta(t))=1-\mu_{1}(\theta(t))
$$

Applying Theorem 4 and set $\alpha=0.3$, the state feedback controller gains are designed as

$$
\begin{aligned}
& K_{1}=[-3.472,5.005,-0.7264] \\
& K_{2}=[-3.215,3.596,-0.6993]
\end{aligned}
$$

Figure 10 shows the state trajectories of the closed-loop truck-trailer system. Figure 11 shows the corresponding control input. These figures demonstrated the effectiveness and feasibility of our proposed methods. 


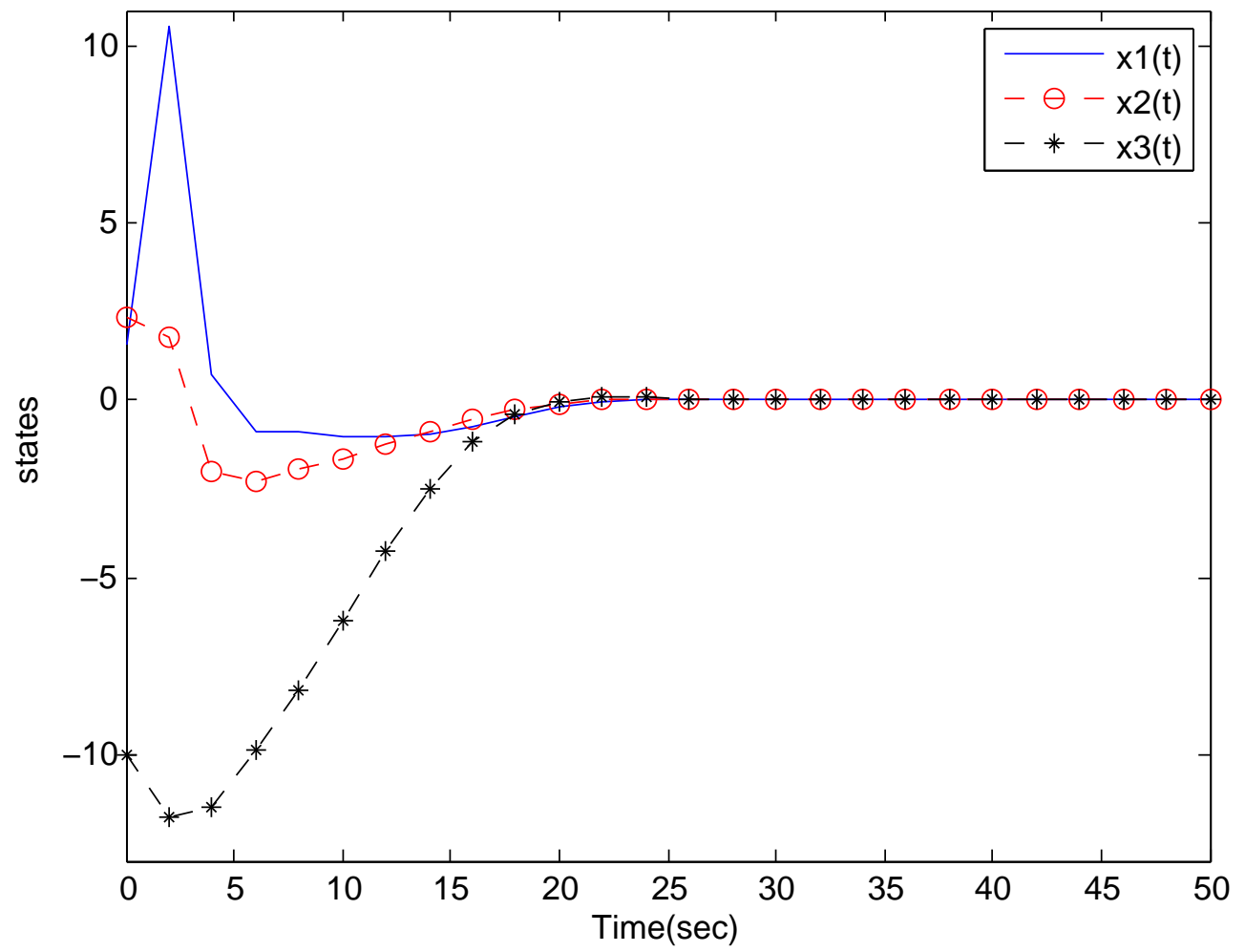

Figure 10: States responses of backing-up truck-trailer 


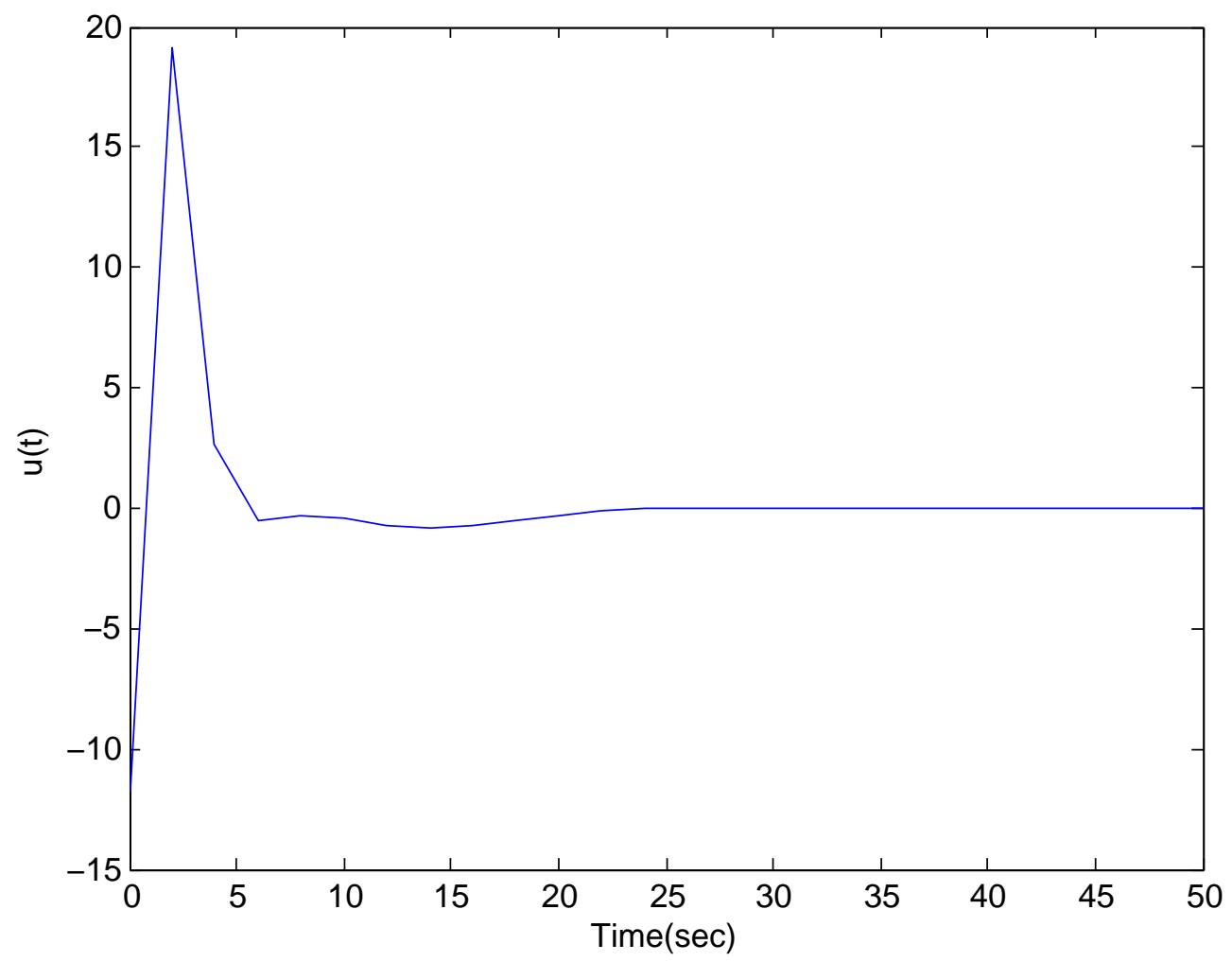

Figure 11: Control input of backing-up truck-trailer

\section{Conclusion}

In this paper, the method of designing state feedback controllers which exponentially stabilize the T-S fuzzy systems was presented. Using guaranteed cost control, PDC controller was designed by minimizing the upper bound of a variation of quadratic performance function which could provide desirable control performance and fast responses simultaneously. Sufficient conditions which guarantee exponential stabilities of the resulting closed-loop systems with predefined convergence rates were proved for continuous- and discretetime T-S fuzzy systems, respectively. The simulation results verified that by 
appropriately setting convergence rate and weighting matrices in the design stage of our method, the response speed of the closed-loop system could be accelerated efficiently and the overall control performance was superior than those of other existing methods, while the control effort was still kept at an acceptable level.

Based on the main results, further works may be carried out, for instance:

1. extensions to deal with more complex nonlinear systems by introducing observers when state-variables are not measurable, as proposed in [35] and $[36]$.

2. taking parameter uncertainty and actuator saturation into considerations, such as non-fragile guaranteed cost control [37] and robust control [38].

\section{Acknowledgment}

This paper is partly supported by the National Science Foundation of China (61473183, 61521063, U1509211), Program of Shanghai Subject Chief Scientist (14XD1402400).

\section{Reference}

[1] H. Wang, K. Tanaka, and M. Griffin, "An approach to fuzzy control of nonlinear systems: stability and design issues," Fuzzy Systems, IEEE Transactions on, vol. 4, no. 1, pp. 14-23, 1996.

[2] K. Tanaka and M. Sugeno, "Stability analysis and design of fuzzy control systems," Fuzzy sets and systems, vol. 45, no. 2, pp. 135-156, 1992. 
[3] Y. Li, S. Tong, and T. Li, "Observer-based adaptive fuzzy tracking control of MIMO stochastic nonlinear systems with unknown control directions and unknown dead zones," Fuzzy Systems, IEEE Transactions on, vol. 23, no. 4, pp. 1228-1241, 2015.

[4] S. Tong, S. Sui, and Y. Li, "Fuzzy adaptive output feedback control of MIMO nonlinear systems with partial tracking errors constrained," Fuzzy Systems, IEEE Transactions on, vol. 23, no. 4, pp. 729-742, 2015.

[5] T. Takagi and M. Sugeno, "Fuzzy identification of systems and its applications to modeling and control," Systems, Man and Cybernetics, IEEE Transactions on, no. 1, pp. 116-132, 1985.

[6] T. Li, S. Tong, and G. Feng, "A novel robust adaptive-fuzzy-tracking control for a class of nonlinear multi-input/multi-output systems," Fuzzy Systems, IEEE Transactions on, vol. 18, no. 1, pp. 150-160, 2010.

[7] Y. Liu, W. Wang, S. Tong, and Y. Liu, "Robust adaptive tracking control for nonlinear systems based on bounds of fuzzy approximation parameters," Systems, Man and Cybernetics, Part A: Systems and Humans, IEEE Transactions on, vol. 40, no. 1, pp. 170-184, 2010.

[8] R. Precup and H. Hellendoorn, "A survey on industrial applications of fuzzy control," Computers in Industry, vol. 62, no. 3, pp. 213-226, 2011.

[9] G. Feng, "A survey on analysis and design of model-based fuzzy control systems," Fuzzy systems, IEEE Transactions on, vol. 14, no. 5, pp. 676697, 2006. 
[10] A. Farahmand and M. Yazdanpanah, "Locally optimal Takagi-Sugeno fuzzy controllers," Decision and Control, European Control Conference, 44th IEEE jointly Conference on, pp. 4095-4099, 2005.

[11] A. Jadbabaie, C. Abdallah, M. Jamshidi, and P. Dorato, "Guaranteedcost control of the nonlinear benchmark problem using model-based fuzzy systems," Control Applications, IEEE International Conference on, vol. 2, pp. 792-796, 1998.

[12] A. Jadbabaie, M. Jamshidi, and A. Titli, "Guaranteed-cost design of continuous-time Takagi-Sugeno fuzzy controllers via linear matrix inequalities," Fuzzy Systems, IEEE International Conference on, vol. 1, pp. 268-273, 1998.

[13] K. Tanaka, T. Ikeda, and H. Wang, "Design of fuzzy control systems based on relaxed LMI stability conditions," Decision and Control, IEEE Conference on, vol. 1, pp. 598-603, 1996.

[14] K. Tanaka, T. Taniguchi, and H. Wang, "Fuzzy control based on quadratic performance function-a linear matrix inequality approach," Decision and Control, IEEE Conference on, vol. 3, pp. 2914-2919, 1998.

[15] S. Wu and C. Lin, "Optimal fuzzy controller design: local concept approach," Fuzzy Systems, IEEE Transactions on, vol. 8, no. 2, pp. 171$185,2000$.

[16] S. Zhou, G. Feng, S. Wu, and C. Lin, "Comment on "Optimal fuzzy controller design: local concept approach" [with reply]," Fuzzy Systems, IEEE Transactions on, vol. 11, no. 2, p. 279, 2003. 
[17] F. Sheikholeslam, "Further comment on "Optimal fuzzy controller design: Local concept approach"," Fuzzy Systems, IEEE Transactions on, vol. 2, no. 16, pp. 544-546, 2008.

[18] M. Athans and P. Falb, Optimal control: an introduction to the theory and its applications. Courier Corporation, 2006.

[19] T. Hu and Z. Lin, Control systems with actuator saturation: analysis and design. Springer Science \& Business Media, 2001.

[20] H. K. Khalil and J. Grizzle, Nonlinear systems, vol. 3. Prentice hall New Jersey, 1996.

[21] K. Tanaka, T. Ikeda, and H. Wang, "Fuzzy regulators and fuzzy observers: relaxed stability conditions and LMI-based designs," Fuzzy Systems, IEEE Transactions on, vol. 6, no. 2, pp. 250-265, 1998.

[22] M. Chilali and P. Gahinet, " $H^{\infty}$ design with pole placement constraints: an LMI approach," Automatic Control, IEEE Transactions on, vol. 41, no. 3, pp. 358-367, 1996.

[23] Z. Han, G. Feng, B. Walcott, and Y. Zhang, " $H^{\infty}$ controller design of fuzzy dynamic systems with pole placement constraints," American Control Conference, pp. 1939-1943, 2000.

[24] C. Li and G. Ma, Optimal control (in Chinese). Science Press, 2011.

[25] C.-H. Fang, Y.-S. Liu, S.-W. Kau, L. Hong, and C.-H. Lee, “A new LMIbased approach to relaxed quadratic stabilization of TS fuzzy control 
systems," Fuzzy Systems, IEEE Transactions on, vol. 14, no. 3, pp. 386397, 2006.

[26] S. Boyd, L. El Ghaoui, E. Feron, and V. Balakrishnan, Linear matrix inequalities in system and control theory, vol. 15. SIAM, 1994.

[27] A. Mosek, "The MOSEK optimization software," Online at http://www. mosek. com, vol. 54, 2010.

[28] J. Löfberg, "Yalmip: A toolbox for modeling and optimization in MATLAB," Computer Aided Control Systems Design, IEEE International Symposium on, pp. 284-289, 2004.

[29] B. Wie and D. Bernstein, "Benchmark problems for robust control design," Journal of Guidance, Control, and Dynamics, vol. 15, no. 5, pp. 1057-1059, 1992.

[30] A. Alleyne, "Physical insights on passivity-based TORA control designs," Control Systems Technology, IEEE Transactions on, vol. 6, no. 3, pp. 436-439, 1998.

[31] L. Hung, H. Lin, and H. Chung, "Design of self-tuning fuzzy sliding mode control for TORA system," Expert Systems with Applications, vol. 32, no. 1, pp. 201-212, 2007.

[32] M. Jankovic, D. Fontaine, and P. Kokotovic, "TORA example: Cascade and passivity control designs," American Control Conference, vol. 6, pp. 4347-4351, 1995. 
[33] K. Tanaka and M. Sano, "A robust stabilization problem of fuzzy control systems and its application to backing up control of a truck-trailer," Fuzzy Systems, IEEE Transactions on, vol. 2, no. 2, pp. 119-134, 1994.

[34] S. Cao, N. Rees, and G. Feng, "Analysis and design for a class of complex control systems part II: fuzzy controller design," Automatica, vol. 33, no. 6, pp. 1029-1039, 1997.

[35] H. Li, Y. Gao, P. Shi, and H.-K. Lam, "Observer-based fault detection for nonlinear systems with sensor fault and limited communication capacity," 2015.

[36] H. Li, C. Wu, S. Yin, and H.-K. Lam, "Observer-based fuzzy control for nonlinear networked systems under unmeasurable premise variables," 2015.

[37] M. Chen and J. Li, "Non-fragile guaranteed cost control for TakagiSugeno fuzzy hyperbolic systems," International Journal of Systems Science, vol. 46, no. 9, pp. 1614-1627, 2015.

[38] L. Zhao and L. Li, "Robust stabilization of T-S fuzzy discrete systems with actuator saturation via PDC and non-PDC law," Neurocomputing, vol. 168, pp. 418-426, 2015. 